\title{
GREEN CURRENTS FOR MEROMORPHIC MAPS OF COMPACT KÄHLER MANIFOLDS
}

\author{
TURGAY BAYRAKTAR
}

\section{INTRODUCTION}

Let $X$ be a compact Kähler manifold and $f: X \rightarrow X$ be a dominant meromorphic map. It is known that we may define a linear pullback map $f^{*}: H^{1,1}(X, \mathbb{R}) \rightarrow$ $H^{1,1}(X, \mathbb{R})$. However, in general this linear action is not compatible with the dynamics of the map $f$. We say that $f$ is 1-regular whenever $\left(f^{n}\right)^{*}=\left(f^{*}\right)^{n}$ for $n=1,2, \ldots$ on $H^{1,1}(X, \mathbb{R})$. In the sequel we will assume that $f$ is 1 -regular. By a standard Perron-Frobenius type argument there exists $\alpha \in H_{\text {psef }}^{1,1}(X, \mathbb{R})$ such that

$$
f^{*} \alpha=\lambda_{1}(f) \alpha
$$

where $\lambda_{1}(f)$ is defined to be the spectral radius of $f^{*}$. Let

$$
H:=\left\{\alpha \in H^{1,1}(X, \mathbb{R}): f^{*} \alpha=\lambda_{1}(f) \alpha\right\}
$$

we also consider

$H_{\mathcal{N}}:=\left\{\alpha \in H^{1,1}(X, \mathbb{R}): \alpha=\lim _{N \rightarrow \infty} \frac{1}{N} \sum_{n=1}^{N} \frac{1}{n^{m-1} \lambda_{1}^{n}}\left(f^{n}\right)^{*} \beta\right.$ for some $\left.\beta \in H_{\text {nef }}^{1,1}(X, \mathbb{R})\right\}$

where $m$ denotes the size of the largest Jordan block associated to $\lambda_{1}(f)$. Then it follows that $H_{\mathcal{N}} \subset H \cap H_{\text {psef }}^{1,1}(X, \mathbb{R})$ and $H_{\mathcal{N}}$ has a non-empty interior in $H$.

In general, a class $\alpha \in H_{\mathcal{N}}$ is not numerically effective (nef). Boucksom [Bou04] has defined the minimal multiplicity $\nu(\alpha, x)$ of a class $\alpha \in H_{\text {psef }}^{1,1}(X, \mathbb{R})$ at a point $x \in X$. This is a local obstruction to the numerical effectiveness of $\alpha \in H_{p s e f}^{1,1}(X, \mathbb{R})$ at $x$. The set

$$
E_{n n}(\alpha):=\{x \in X: \nu(\alpha, x)>0\}
$$

is called the non-nef locus of $\alpha$. A property of $E_{n n}(\alpha)$ is that if $C \subset X$ is an irreducible algebraic curve such that $\alpha \cdot C<0$ then $C \subset E_{n n}(\alpha)$. We let $I_{f^{k}}$ denote the indeterminacy locus of the iterate $f^{k}$.

Theorem 1.1. Let $f: X \rightarrow X$ be a dominant meromorphic map. If $f$ is 1-regular and $\lambda_{1}(f)>1$ then $E_{n n}(\alpha) \subset \bigcup_{k=1}^{\infty} I_{f^{k}}$ for every $\alpha \in H_{\mathcal{N}}$.

As a corollary we obtain that every curve $C$ such that $\alpha \cdot C<0$ is a subset of

$\bigcup_{k=1}^{\infty} I_{f^{k}}$. Moreover, the non-nef locus $E_{n n}(\alpha)$ does not contain any hypersurface of $X$.

Many authors have constructed positive closed invariant currents to represent

Date: February 9, 2012. 
the invariant classes. These constructions, however, assume that the class is nef or sometimes even Kähler. Here we consider some cases where the invariant class is merely psef.

Let us fix a smooth representative $\theta \in \alpha$. We say that an upper semi-continuous function $\phi \in L^{1}(X)$ is a $\theta$-psh function if $\theta+d d^{c} \phi \geq 0$ in the sense of currents. Following [DPS01] we define

$$
v_{\alpha}^{\min }:=\sup \{\phi \leq 0: \phi \text { is } \theta \text {-psh function }\} .
$$

Thus, $\theta+d d^{c} v_{\alpha}^{\min } \in \alpha$ is a positive closed $(1,1)$ current with minimal singularities.

Theorem 1.2. Let $f: X \rightarrow X$ be a 1-regular dominant meromorphic map and $\alpha \in H_{p s e f}^{1,1}(X, \mathbb{R})$ such that $f^{*} \alpha=\lambda \alpha$ for some $\lambda>1$. If

$$
\frac{1}{\lambda^{n}} v_{\alpha}^{\text {min }} \circ f^{n} \rightarrow 0 \text { in } L^{1}(X)
$$

then for every smooth form $\theta \in \alpha$ we have the existence of the limit

$$
T_{\alpha}:=\lim _{n \rightarrow \infty} \frac{1}{\lambda^{n}}\left(f^{n}\right)^{*} \theta
$$

which depends only on the class $\alpha . T_{\alpha}$ is a positive closed $(1,1)$ current satisfying $f^{*} T_{\alpha}=\lambda T_{\alpha}$. Furthermore,

(1) $T_{\alpha}$ is minimally singular among the invariant currents which belong to the class $\alpha$.

(2) $T_{\alpha}$ is extreme within the cone of positive closed $(1,1)$ currents whose cohomology class belongs to $\mathbb{R}^{+} \alpha$.

We have seen that such $\alpha$ exists for $\lambda=\lambda_{1}(f)$ but we can also allow other values of $\lambda$ as well. We also prove that $(\star)$ is a necessary condition under natural dynamical assumptions (see Proposition 4.3).

The following result provides and algebraic criterion for the existence of Green currents when $X$ is projective:

Theorem 1.3. Let $X$ be a projective manifold and $f: X \rightarrow X$ be a dominant 1regular rational map. Assume that $\lambda:=\lambda_{1}(f)>1$ is a simple eigenvalue of $f^{*}$ with $f^{*} \alpha_{f}=\lambda \alpha_{f}$. If $\alpha_{f} \cdot C \geq 0$ for every algebraic irreducible curve $C \subset E_{f}^{-}:=f\left(I_{f}\right)$ then

$$
\frac{1}{\lambda^{n}} v_{\alpha}^{\min } \circ f^{n} \rightarrow 0 \text { in } L^{1}(X) .
$$

In the last part of this work, we present some examples of birational maps in higher dimensions which fall into the frame work of Theorem 1.2, nevertheless the invariant class is not nef.

Let $f:=L \circ J$ where $J: \mathbb{P}^{d} \rightarrow \mathbb{P}^{d}$

$$
J\left[x_{0}: x_{1}: \cdots: x_{d}\right]=\left[x_{0}^{-1}: x_{1}^{-1}: \cdots: x_{d}^{-1}\right]
$$

and $L$ is a linear map given by given by a $(d+1) \times(d+1)$ matrix of the form

$$
L=\left[\begin{array}{ccccc}
a_{0}-1 & a_{1} & a_{2} & \ldots & a_{d} \\
a_{0} & a_{1}-1 & a_{2} & \ldots & a_{d} \\
a_{0} & a_{1} & a_{2}-1 & \ldots & a_{d} \\
\vdots & \vdots & \vdots & \ddots & \vdots \\
a_{0} & a_{1} & a_{2} & \ldots & a_{d}-1
\end{array}\right]
$$


with $a_{j} \in \mathbb{C}$ and $\sum_{j=0}^{d} a_{j}=2$. The linear map $L$ is involutive that is $L=L^{-1}$ in $P G L(d+1, \mathbb{C})$. Let $\Sigma_{i}:=\left\{\left[x_{0}: \cdots: x_{d}\right] \in \mathbb{P}^{d}: x_{i}=0\right\}$ then $p_{i}:=f\left(\Sigma_{i}\right) \in \mathbb{P}^{d}$ is the $i^{t h}$ column of the matrix $L$. We define its orbit $\mathcal{O}_{i}$ as follows: $\mathcal{O}_{i}=$ $\left\{p_{i}, f\left(p_{i}\right), f^{2}\left(p_{i}\right), \ldots, f^{N_{i}-1}\left(p_{i}\right)\right\}$ if $f^{j}\left(p_{i}\right) \notin I_{f}$ for $0 \leq j \leq N_{i}-2$ and $f^{N_{i}-1}\left(p_{i}\right) \in$ $I_{f}$ for some $N_{i} \in \mathbb{N}$, otherwise $\mathcal{O}_{i}=\left\{p_{i}, f\left(p_{i}\right), f^{2}\left(p_{i}\right), \ldots\right\}$. If $\mathcal{O}_{i}$ is finite (the first case above) we say that the orbit of $\Sigma_{i}$ is singular of length $N_{i}$. It follows from BK04 that there exists a complex manifold $X$ together with a proper modification $\pi: X \rightarrow \mathbb{P}^{d}$ such that the induced map $f_{X}: X \rightarrow X$ is 1-regular. Moreover, if the length of the singular orbits are long enough (see Theorem 6.1) then $\lambda_{1}(f)>1$ is the unique simple eigenvalue of $\left.f^{*}\right|_{H^{1,1}(X, \mathbb{R})}$ of modulus greater than one. We define $S:=\left\{i \in\{0,1, \ldots, d\} \mid \mathcal{O}_{i}\right.$ is singular $\}$ and denote its cardinality by $|S|$. If $S$ is non-empty, by conjugating $f$ with an involution without lost of generality we may assume that $S=\{0, \ldots, k\}$.

Theorem 1.4. Let $f_{X}: X \rightarrow X$ be as above with $\lambda:=\lambda_{1}\left(f_{X}\right)>1$ and $\alpha_{f} \in$ $H_{p s e f}^{1,1}(X, \mathbb{R})$ such that $f^{*} \alpha_{f}=\lambda \alpha_{f}$. Then $\alpha_{f}$ is nef if and only if $|S| \leq 1$. Moreover, if $2 \leq|S| \leq d$ and all singular orbits of $f$ have the same length then

$$
E_{n n}\left(\alpha_{f}\right)= \begin{cases}\left\{\left[x_{0}: x_{1}: \cdots: x_{d}\right]: x_{i}=0 \text { for } k+1 \leq i \leq d\right\} & \text { if } k \leq d-2 \\ \bigcup_{i=0}\left\{\left[x_{0}: x_{1}: \cdots: x_{d}\right]: x_{i}=x_{d}=0\right\} & \text { if } k=d-1\end{cases}
$$

We also show that these maps fall into frame work of Theorem 1.2:

Theorem 1.5. Let $f_{X}: X \rightarrow X$ be as above with $\lambda_{1}\left(f_{X}\right)>1$. If $a_{i} \neq 0$ for every $i \in S$ then condition ( $\star$ ) in Theorem 1.2 holds.

The outline of the paper as follows. In section 2 , we provide the basic definitions and results which we will use in the sequel. In section 3, we discuss invariance properties of closed convex cones in $H^{1,1}(X, \mathbb{R})$ and prove Theorem 1.1. Section 4 is devoted to the proof of Theorem 1.2. We also discuss some cases for which $(\star)$ holds in section 4 . In section 5 , we discuss rational maps and prove Theorem 1.3. In the last section, we prove Theorems 1.4 and 1.5 .

\section{ACKNOWLEDGEMENT}

I would like to express my sincere thanks to my advisor E. Bedford for his guidance and interest on this work. I am also grateful to J. Diller and V. Guedj for their comments and suggestions on an earlier draft. I also thank to the referee for his suggestions.

\section{Preliminaries}

2.1. Positive Cones. Let $X$ be a compact Kähler manifold of dimension $k$ and $\omega$ be a fixed Kähler form satisfying $\int_{X} \omega^{k}=1$. All volumes will be computed with respect to the probability volume form $d V:=\omega^{k}$. Let $H^{1,1}(X)$ denote the Dolbeault cohomology group and let $H^{2}(X, \mathbb{Z}), H^{2}(X, \mathbb{R})$ and $H^{2}(X, \mathbb{C})$ denote the de-Rham cohomology groups with coefficients in $\mathbb{Z}, \mathbb{R}, \mathbb{C}$. We also set

$$
H^{1,1}(X, \mathbb{R}):=H^{1,1}(X) \cap H^{2}(X, \mathbb{R}) .
$$


Definition 2.1. A class $\alpha \in H^{1,1}(X, \mathbb{R})$ is called Kähler if $\alpha$ can be represented by a Kähler form. We denote the set of all Kähler classes by $\mathcal{K}$. A class $\alpha$ is called numerically effective (nef) if it lies in the closure of the Kähler cone. The set of all nef classes will be denoted by $H_{n e f}^{1,1}(X, \mathbb{R})$.

An upper semi continuous function $\varphi \in L^{1}(X)$ is called quasi-plurisubharmonic (qpsh) if there exists a smooth closed form $\theta$ such that $\theta+d d^{c} \varphi \geq 0$ in the sense of currents. Notice that a qpsh function is locally sum of a smooth function and a psh function. A closed $(1,1)$ current $\mathrm{T}$ is called almost positive if there exists a real smooth $(1,1)$ form $\gamma$ such that $T \geq \gamma$.

The Lelong number of a positive closed $(1,1)$ current $T$ is defined by

$$
\nu(T, x):=\liminf _{z \rightarrow x} \frac{\phi(z)}{\log |x-z|}
$$

where $\phi$ is a local potential for $T$ that is $T=d d^{c} \phi$ near $x$. This definition is independent of the choice of the potential $\phi$ and the local coordinates. If $T$ is almost positive then the Lelong numbers are still well-defined since the negative part contributes for zero. It follows from a theorem of Thie that $\nu([D], x)=$ mult $_{x} D$ where $[D]$ is the current of integration along an effective divisor and mult $_{x}$ is the multiplicity of $D$ at $x$. We denote the sub-level sets by $E_{c}(T):=\{x \in X: \nu(T, x) \geq$ $c\}$. A Theorem of Siu asserts that $E_{c}(T)$ is an analytic set of codimension at least 1. We also set

$$
E_{+}(T):=\cup_{c>0} E_{c}(T) .
$$

A class $\alpha \in H^{1,1}(X, \mathbb{R})$ is called pseudo-effective (psef) if there exists a positive closed $(1,1)$ current $T$ such that $\{T\}=\alpha$. The set of all psef classes, $H_{p s e f}^{1,1}(X, \mathbb{R})$ is a closed convex cone containing $H_{n e f}^{1,1}(X, \mathbb{R})$. A positive closed current $T$ is called Kähler if there exists small $\epsilon>0$ such that $T \geq \epsilon \omega$. A class $\alpha \in H^{1,1}(X, \mathbb{R})$ is said to be big if there exists a Kähler current $T$ such that $\alpha=\{T\}$. We denote the set of all big classes by $H_{b i g}^{(1,1)}(X, \mathbb{R})$. This is an open convex cone and coincides with the interior of $H_{\text {psef }}^{1,1}(X, \mathbb{R})$. Finally, we stress that these definitions coincide with the classical ones in complex geometry [Dem92].

Theorem 2.2 ([Bou04, DP04]). A class $\alpha \in H_{\text {nef }}^{1,1}(X, \mathbb{R})$ is big if and only if $\alpha^{n} \neq 0$.

2.2. Currents with analytic singularities. Following Dem92] and Bou04, a closed almost positive $(1,1)$ current $T=\theta+d d^{c} \phi$ is said to have analytic singularities along a subscheme $V(\mathcal{I})$ defined by a coherent ideal sheaf $\mathcal{I}$ if there exists $c>0$ and locally

$$
\phi=\frac{c}{2} \log \left(\left|f_{1}\right|^{2}+. .+\left|f_{N}\right|^{2}\right)+u
$$

where $u$ is a smooth function and $f_{1}, . ., f_{N}$ 's are holomorphic functions which are local generators of $\mathcal{I}$. Blowing-up $X$ along $V(\mathcal{I})$ and resolving the singularities in the sense of Hironaka, we obtain a modification $\mu: \widetilde{X} \rightarrow X$. Moreover, $D:=\mu^{-1}(V(\mathcal{I}))$ is an effective divisor in $\widetilde{X}$ and $\mu^{*} T$ has analytic singularities along $D$, thus it follows from Siu decomposition that

$$
\mu^{*} T=\theta+c D
$$

where $\theta$ is a smooth $(1,1)$ form. Furthermore, if $T \geq \gamma$ then we have $\theta \geq \mu^{*} \gamma$. In particular, if $T \geq 0$ then $\theta \geq 0$. This decomposition is called $\log$ resolution of singularities of $T$. 
Theorem 2.3 (Dem92]). Let $T \geq \gamma$ be an almost positive closed $(1,1)$ current on $X$. Then there exists a sequence of positive real numbers $\epsilon_{n}$ decreasing to 0 and a sequence of almost positive closed $(1,1)$ currents $T_{n} \in\{T\}$ with analytic singularities such that $T_{n} \rightarrow T$ weakly, $T_{n} \geq \gamma-\epsilon_{n} \omega$ and $\nu\left(T_{n}, x\right)$ increases uniformly to $\nu(T, x)$ with respect to $x \in X$.

2.3. Currents with minimal singularities. Let $\varphi_{1}$ and $\varphi_{2}$ be two qpsh functions. Following DPS01, we say that $\varphi_{1}$ is less singular than $\varphi_{2}$ if $\varphi_{2} \leq \varphi_{1}+C$ for some constant $C$. If $T_{1}$ and $T_{2}$ are two closed almost positive currents we write $T_{i}=\theta_{i}+d d^{c} \varphi_{i}$ where $\theta_{i} \in\left\{T_{i}\right\}$ is a smooth closed form and $\varphi_{i}$ is a qpsh function. We say that $T_{1}$ is less singular than $T_{2}$ if $\varphi_{2} \leq \varphi_{1}+C$. Notice that this definition is independent of the choice of the representatives $\theta_{i}$ and potentials $\varphi_{i}$.

For a class $\alpha \in H^{1,1}(X, \mathbb{R})$ and a real smooth $(1,1)$ form $\gamma$, we denote the set of all closed almost positive $(1,1)$ currents $T \in \alpha$ satisfying $T \geq \gamma$ by $\alpha[\gamma]$. We fix a smooth represantative $\theta \in \alpha$ and define

$$
v_{\alpha, \gamma}^{\min }:=\sup \left\{\varphi \leq 0 \mid \theta+d d^{c} \varphi \geq \gamma\right\}
$$

It follows that $T_{\alpha, \gamma}^{\min }:=\theta+d d^{c} v_{\alpha, \gamma}^{\min } \in \alpha[\gamma]$ and $\nu\left(T_{\alpha, \gamma}^{\min }, x\right) \leq \nu(T, x)$ for every $x \in X$ and for every $T \in \alpha[\gamma]$. If, in particular $\gamma=0$ then we write $T_{\alpha, \gamma}^{\min }=T_{\alpha}^{\min }$ and refer to it as the minimally singular current. Notice that minimally singular currents are not unique in general. For example, if $\alpha \in \mathcal{K}$ then every smooth positive closed form $\theta \in \alpha$ is a minimally singular current. However, if $S=\theta^{\prime}+d d^{c} u_{\alpha, \gamma}^{\min } \in \alpha$ is another such current, since $v_{\alpha, \gamma}^{\min }-u_{\alpha, \gamma}^{\min } \in L_{l o c}^{1}(X)$ and $d d^{c}\left(v_{\alpha, \gamma}^{\min }-u_{\alpha, \gamma}^{\min }\right)$ is smooth, $v_{\alpha, \gamma}^{\min }-u_{\alpha, \gamma}^{\min }$ is also smooth and hence, bounded. Thus, $v_{\alpha, \gamma}^{\min }$ and $u_{\alpha, \gamma}^{\min }$ are equivalent in the sense of singularities. Therefore, for a fixed class $\alpha \in H_{\text {psef }}^{1,1}(X, \mathbb{R})$, the current of minimal singularities is well-defined modulo $d d^{c}\left(C^{\infty}\right)$.

2.4. Minimal multiplicities and non-nef locus. Following Bou04, for a class $\alpha \in H_{\text {psef }}^{1,1}(X, \mathbb{R})$ we define

$$
\nu(\alpha, x):=\sup _{\epsilon>0} \nu\left(T_{\alpha, \epsilon}^{\min }, x\right)
$$

where $T_{\alpha, \epsilon}^{\min }:=T_{\alpha,-\epsilon \omega}^{\min } \in \alpha[-\epsilon \omega]$. Since the right hand side in the definition of $\nu(\alpha, x)$ is increasing as $\epsilon$ decreases, the sup coincides with the limit. This definition is independent of the choice of the Kähler form $\omega$. We also remark that for every positive closed $(1,1)$ current $T \in \alpha$ and $x \in X$, we have $0 \leq \nu(\alpha, x) \leq \nu(T, x) \leq C$ where $C>0$ is a constant depending only on the cohomology class $\alpha$.

If $A$ is an analytic subset of $\mathrm{X}$ we define

$$
\nu(\alpha, A):=\inf _{x \in A} \nu(\alpha, x) .
$$

A class $\alpha \in H_{\text {psef }}^{1,1}(X, \mathbb{R})$ is called nef in codimension 1 if $\nu(\alpha, D)=0$ for every prime divisor $D \subset X$. We denote the set of all such classes by $\mathcal{E}_{1}$. It follows from the following proposition that $\mathcal{E}_{1} \subset H_{\text {psef }}^{1,1}(X, \mathbb{R})$ is also a closed convex cone.

Proposition 2.4 ([Bou04]). Let $\alpha \in H_{\text {psef }}^{1,1}(X, \mathbb{R})$ be a class

(i) $\alpha$ is nef if and only if $\nu(\alpha, x)=0$ for every $x \in X$.

(ii) $\alpha \rightarrow \nu(\alpha, x)$ is sub-additive and homogenous in $\alpha$ for every $x \in X$.

(iii) $\alpha \rightarrow \nu(\alpha, x)$ is lower semi-continuous on $H_{\text {psef }}^{1,1}(X, \mathbb{R})$ and continuous on $H_{\text {big }}^{1,1}(X, \mathbb{R})$ for every $x \in X$. 
(iv) If $\alpha \in H_{b i g}^{1,1}(X, \mathbb{R})$ then $\nu(\alpha, x)=\nu\left(T_{\alpha}^{\text {min }}, x\right)$ for every $x \in X$.

Corollary 2.5. If $\alpha \in H_{\text {nef }}^{1,1}(X, \mathbb{R}) \cap H_{\text {big }}^{1,1}(X, \mathbb{R})$ then $\nu\left(T_{\alpha}^{\text {min }}, x\right)=0$ for every $x \in X$. Moreover, if $\alpha \in H_{\text {psef }}^{1,1}(X, \mathbb{R})$ and $\nu\left(T_{\alpha}^{\text {min }}, x\right)=0$ for every $x \in X$ then $\alpha$ is nef.

Definition 2.6 ([Bou04] $)$. Let $\alpha \in H_{\text {psef }}^{1,1}(X, \mathbb{R})$ then non-nef locus of $\alpha$ is defined by

$$
E_{n n}(\alpha):=\{x \in X \mid \nu(\alpha, x)>0\} .
$$

We also have the following description of the non-nef locus:

Proposition 2.7. Let $\alpha \in H_{\text {psef }}^{1,1}(X, \mathbb{R})$ then

$$
E_{n n}(\alpha)=\bigcup_{\epsilon>0} \bigcap_{T} \mu(|D|)
$$

where $T$ runs over the set $\{T \in \alpha[-\epsilon \omega]: T$ has analytic singularities $\}$ and $\mu$ : $\widetilde{X} \rightarrow X, \mu^{*} T=\theta+[D]$ is log resolution of singularities of $T$ and $|D|$ denotes the support of the current of integration $[D]$.

Proof. Let $x \in \bigcup_{\epsilon>0} \bigcap_{T} \mu(|D|)$. Then there exists $\epsilon_{1}>0$ such that $x \in \mu(|D|)$ for every $T \in \alpha\left[-\epsilon_{1} \omega\right]$ which has analytic singularities and $\log$ resolution $\mu: \widetilde{X} \rightarrow X$, $\mu^{*} T=\theta+[D]$. This implies that $\nu(T, x)>0$. Let $\epsilon<\epsilon_{1}$ and let $T_{\alpha, \epsilon}^{\min }$ be current of minimal singularities. By Theorem 2.3 there exists a sequence $T_{k} \in \alpha\left[-\epsilon_{1} \omega\right]$ with analytic singularities such that $T_{k}$ converges weakly to $T$ and $\nu\left(T_{k}, x\right)$ increases to $\nu\left(T_{\alpha, \epsilon}^{\min }, x\right)$. Thus, $0<\nu\left(T_{\alpha, \epsilon}^{\min }, x\right) \leq \nu(\alpha, x)$. That is $x \in E_{n n}(\alpha)$.

To prove reverse inclusion, let $x \in E_{n n}(\alpha)$, then by definition of $\nu(\alpha, x)$ there exists $\epsilon>0$ such that $0<\nu\left(T_{\alpha, \epsilon}^{\min }, x\right)$. Now, let $T \in \alpha[\epsilon]$ has analytic singularities. Resolving it's singularities we obtain $\mu: \widetilde{X} \rightarrow X$ such that $\mu^{*} T=\theta+[D]$ where $\theta \geq-\epsilon \omega$ is smooth and $D$ is an effective divisor. Since $0<\nu\left(T_{\alpha, \epsilon}^{\min }, x\right) \leq \nu(T, x) \leq$ $\nu\left(\mu^{*}(T), p\right)$ for every $p \in \widetilde{X}$ with $\mu(p)=x$, we conculde that $x \in \mu(|D|)$.

Definition 2.8. Let $\alpha \in H_{\text {psef }}^{1,1}(X, \mathbb{R})$ be a class. An irreducible algebraic curve $C$ is called $\alpha$-negative if the intersection product $\alpha \cdot C<0$.

Proposition 2.9 ([BDPP $)$. For $\alpha \in H_{\text {psef }}^{1,1}(X, \mathbb{R})$ every $\alpha$-negative curve $C$ is contained in $E_{n n}(\alpha)$.

Proof. If $C \not \subset E_{n n}(\alpha)$ then for every $\epsilon>0$ there exists $T \in \alpha[-\epsilon \omega]$ with analytic singularities such that $C \not \subset \mu(|D|)$ where $\mu: \widetilde{X} \rightarrow X$ is $\log$ reolution of $T$ and $\mu^{*}(T)=\theta+[D]$.

Let $\widetilde{C} \subset \widetilde{X}$ be the strict transform of $C$ so that $\mu_{*} \widetilde{C}=C$ and define $S=T+\epsilon \omega \geq$ 0 . Then $\mu^{*} S=\theta_{\epsilon}+[D]$ where $\theta_{\epsilon} \geq 0$ since $S \geq 0$. Thus,

$$
\alpha+\epsilon\{\omega\} \cdot C=\left\langle S, \mu_{*} \widetilde{C}\right\rangle=\left\langle\mu^{*} S, \widetilde{C}\right\rangle=\left\langle\theta_{\epsilon}+[D], \widetilde{C}\right\rangle \geq 0
$$

the last inequality follows from $\theta_{\epsilon} \geq 0$ and $\widetilde{C} \not \subset D$. Since $\epsilon>0$ is arbitrary we obtain $\alpha \cdot C \geq 0$.

Remark 2.10. Thus, the non-nef locus contains the union of the $\alpha$-negative curves. However, in general, the non-nef locus of a pseudo-effective class $\alpha$ is not equal to union of $\alpha$-negative curves (see [BDPP]). 


\section{Dynamics of Meromorphic maps}

Let $X$ be a compact Kähler manifold of dimension $k$ and $f: X \rightarrow X$ be a meromorphic map that is $f$ is holomorphic on the set $X \backslash I_{f}$ such that the closure of the graph $\Gamma_{f}$ of $f: X \backslash I_{f} \rightarrow X$ in $X \times X$ is a irreducible analytic set of dimension $k$. Let $\pi_{i}: X \times X \rightarrow X$ denote the canonical projections. Then $I_{f}$ coincides with the set of points $z$ for which $\pi_{1}^{-1}(z) \cap \Gamma_{f}$ contains more than one point. The set $I_{f}$, called the indeterminacy set of $f$, is also an analytic set of codimension at least 2. In fact, for every $z \in I_{f}$ the set $\pi_{1}^{-1}(z) \cap \Gamma_{f}$ has positive dimension. Moreover, $X \backslash I_{f}$ is the largest open set where $f$ is holomorphic. We also set

$$
\mathcal{I}_{\infty}:=\bigcup_{n \geq 1} I_{f^{n}} .
$$

For a subset $Z \subset X$ we define the total transform of $Z$ under $f$ by

$$
f(Z):=\pi_{2}\left(\pi_{1}^{-1}(Z) \cap \Gamma_{f}\right) .
$$

With the above convention we define $E_{f}^{-}:=f\left(I_{f}\right)$.

We say that $f$ is dominant if the projection $\pi_{2}$ restricted to $\Gamma_{f}$ is surjective. This is equivalent to saying that Jacobian determinant of $f$ does not vanish identically in any coordinate chart. We refer the reader to the surveys [Sib99] and Gue for basic properties of meromorphic maps.

It's well known that $f$ induces a linear action on $H^{p, p}(X, \mathbb{R})$ as follows: Let $\widetilde{\Gamma_{f}}$ denote a desingularization of $\Gamma_{f}$ then the diagram

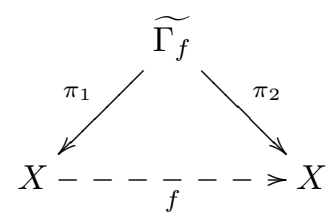

commutes . Let $\theta$ be a smooth closed $(p, p)$ form on $\mathrm{X}$ then we set $f^{*} \theta=\left(\pi_{1}\right)_{*}\left(\pi_{2}\right)^{*} \theta$ where $\left(\pi_{2}\right)^{*} \theta$ is a smooth form and the later is push-forward as a current. Since pullback and push-forward commute with $d$ operator, $f^{*} \theta$ is a $d$-closed $(p, p)$ current. Then we set

$$
f^{*}\{\theta\}=\left\{f^{*} \theta\right\}
$$

where $\{\theta\}$ denotes de-Rham cohomology class of $\theta$ and $\left\{f^{*} \theta\right\}$ is the de-Rham cohomology class of $f^{*} \theta$.

Similarly, one can define push-forward by $f_{*} \theta=\left(\pi_{2}\right)_{*}\left(\pi_{1}\right)^{*} \theta$. It follows that the action of pull-back on $H^{p, p}(X, \mathbb{R})$ is dual to that of push-forward on $H^{k-p, k-p}(X, \mathbb{R})$ with respect to intersection product.

We say that $f$ is $p$-regular whenever $\left(f^{n}\right)^{*}=\left(f^{*}\right)^{n}$ on $H^{p, p}(X, \mathbb{R})$ as linear maps for $n=1,2, \ldots$

We also denote

$$
\delta_{p}(f):=\int_{X \backslash I_{f}} f^{*} \omega^{p} \wedge \omega^{k-p}
$$

and the $p^{\text {th }}$ dynamical degree of $f$ by

$$
\lambda_{p}(f):=\limsup _{n \rightarrow \infty}\left[\delta_{p}\left(f^{n}\right)\right]^{\frac{1}{n}} .
$$

In particular, if $f$ is p-regular then $\lambda_{p}(f)$ coincides with the spectral radius of $\left.f^{*}\right|_{H^{p, p}(X, \mathbb{R})}$. 
3.1. Invariant Classes and Singularities. We denote the set of all positive closed $(1,1)$ currents by $\mathcal{T}(X)$. Following Gue02 we define the pull-back of $T$ as follows: we fix a point $x_{0} \in X \backslash I_{f}$, then locally we can write $T=d d^{c} u$ for a psh function $u$ near $f\left(x_{0}\right)$ and define $f^{*} T=d d^{c} u \circ f$ near $x_{0}$ which is independent of the choice of the local potential $u$. Therefore, we obtain a well-defined positive closed $(1,1)$ current on $X \backslash I_{f}$. Now, since $I_{f}$ is an analytic set of codimension at least two it follows form [HP75] that it extends trivially to a unique positive closed $(1,1)$ current $f^{*} T$ on $X$. Furthermore, since $f$ is dominant the action $T \rightarrow f^{*} T$ is continuous with respect to weak topology on postive closed $(1,1)$ currents (Meo96]). Moreover, $\left\{f^{*} T\right\} \in H^{1,1}(X, \mathbb{R})$ is independent of the choice of $T \in \alpha$ for $\alpha \in H_{p s e f}^{1,1}(X, \mathbb{R})$ and $f^{*} \alpha=\left\{f^{*} T\right\} \in H_{\text {psef }}^{1,1}(X, \mathbb{R})$. Notice that $H_{p s e f}^{1,1}(X, \mathbb{R})$ is a closed, convex cone which is strict (i.e. $\left.H_{p s e f}^{1,1}(X, \mathbb{R}) \cap-H_{\text {psef }}^{1,1}(X, \mathbb{R})=\{0\}\right)$. Since $H_{\text {psef }}^{1,1}(X, \mathbb{R})$ is invariant under the linear action $f^{*}: H^{1,1}(X, \mathbb{R}) \rightarrow H^{1,1}(X, \mathbb{R})$ it follows from a Perron-Frobenius type argument ([DF01] ) that there exists a class $\alpha \in H_{p s e f}^{1,1}(X, \mathbb{R})$ such that $f^{*} \alpha=r_{1}(f) \alpha$ where $r_{1}(f)$ is the spectral radius of $\left.f^{*}\right|_{H^{1,1}(X, \mathbb{R})}$. In particular, if $f$ is 1-regular then $r_{1}(f)=\lambda_{1}(f)$.

The following argument is adapted from [DS05]. We choose a basis for $H^{1,1}(X, \mathbb{C})=$ $H^{1,1}(X, \mathbb{R}) \otimes_{\mathbb{R}} \mathbb{C}$ so that the associated matrix of $f^{*}$ is in Jordan form. Let $J_{\lambda_{j}, m_{j}}$ denote its Jordan blocks for $1 \leq j \leq r$. In other words, we can decompose $H^{1,1}(X, \mathbb{C})$ into a direct sum of complex subspaces $E_{j}$

$$
H^{1,1}(X, \mathbb{C})=\bigoplus_{1 \leq j \leq r} E_{j} \text { with } \operatorname{dim} E_{j}=m_{j} \text { and } \sum_{j=1}^{r} m_{j}=h^{1,1}
$$

such that the restriction of $f^{*}$ to $E_{j}$ is given by the Jordan block $J_{\lambda_{j}, m_{j}}$. Since $f^{*}$ preserves the psef cone which is a proper cone, we may assume that $\lambda_{1}=r_{1}(f)$ and $m:=m_{1}$ is the index of the spectral radius. Moreover, we can also assume that $\left(\left|\lambda_{j}\right|, m_{j}\right)$ is ordered so that either $\left|\lambda_{j}\right|>\left|\lambda_{j+1}\right|$ or $\left|\lambda_{j}\right|=\left|\lambda_{j+1}\right|$ and $m_{j} \geq m_{j+1}$ for $1 \leq j \leq r$. Let $\nu$ be the integer such that $\left|\lambda_{j}\right|=\lambda_{1}$ for $1 \leq j \leq \nu$. Let $\tilde{E}_{j}$ denote the hyperplane generated by the first $m_{j}-1$ vectors of the basis of $E_{j}$ associated to the Jordan form. Then we have $\left\|\left(f^{*}\right)^{n} v\right\| \sim n^{m-1} \lambda_{1}^{n}$ for every $v \notin \tilde{E}_{1} \oplus \cdots \oplus \tilde{E}_{\nu} \oplus E_{\nu+1} \oplus \cdots \oplus E_{r}$. Notice that this property holds for every $v \in \mathcal{K}$ because given any $v^{\prime} \in H^{1,1}(X, \mathbb{R})$ we can find $v^{\prime \prime} \in \mathcal{K}$ and $\sigma \geq 0$ such that $v^{\prime}=\sigma v-v^{\prime \prime}$. We let $F_{j}$ denote the eigenspace of $f_{\left.\right|_{E_{j}}}^{*}$, which is a complex line. We define

$$
F^{\mathbb{C}}:=F_{1} \oplus \cdots \oplus F_{\nu} \text { and } H^{\mathbb{C}}:=\bigoplus_{\substack{\lambda_{j}=\lambda_{1} \\ m_{j}=m}} F_{j} .
$$

We also set $F:=F^{\mathbb{C}} \cap H^{1,1}(X, \mathbb{R})$ and $H:=H^{\mathbb{C}} \cap H^{1,1}(X, \mathbb{R})$. Notice that for any $2 \leq j \leq \nu$ there exists a unique $\theta_{j} \in \mathbb{S}:=\mathbb{R} / 2 \pi \mathbb{Z}$ such that $\lambda_{j}=\lambda_{1} \exp \left(i \theta_{j}\right)$. Let $\theta:=\left(\theta_{2}, \ldots, \theta_{\nu}\right) \in \mathbb{S}^{\nu-1}$. We let $\Theta$ denote the closed subgroup of $\mathbb{S}^{\nu-1}$ generated by $\theta$. This is a finite union of real tori. The orbit of each point $\theta^{\prime} \in \Theta$ under the translation $\theta^{\prime} \rightarrow \theta^{\prime}+\theta$ is dense in $\Theta$. If $\lambda_{j}=\lambda_{1}$ for every $2 \leq j \leq \nu$ then $F=H$ and $\Theta=\{0\}$. We also set

$$
\Lambda_{N}:=\frac{1}{N} \sum_{n=1}^{N} \frac{\left(f^{*}\right)^{n}}{n^{m-1} \lambda_{1}^{n}}
$$

For the proof of the following proposition we refer the reader to [DS05]. 
Proposition 3.1. Assume that $\lambda_{1}>1$. Then the sequence $\left(\Lambda_{N}\right)$ converges to a surjective real linear map $\Lambda_{\infty}: H^{1,1}(X, \mathbb{R}) \rightarrow H$. Let $n_{i}$ be an increasing sequence of positive integers then $\left(n_{i}^{1-m} \lambda_{1}^{-n_{i}}\left(f^{*}\right)^{n_{i}}\right)$ converges if and only if $\left(n_{i} \theta\right)$ converges. Moreover, any limit $L_{\infty}$ of $\left(n_{i}^{1-m} \lambda_{1}^{-n_{i}}\left(f^{*}\right)^{n_{i}}\right)$ is a surjective real linear map $L_{\infty}: H^{1,1}(X, \mathbb{R}) \rightarrow F$.

We also define

$$
H_{p s e f}:=H \cap H_{p s e f}^{1,1}(X, \mathbb{R})
$$

and

$$
H_{\mathcal{N}}:=\Lambda_{\infty}\left(H_{n e f}^{1,1}(X, \mathbb{R})\right)
$$

It's clear that $H_{\mathcal{N}} \subset H_{\text {psef }}$. Moreover, it follows from Proposition 3.1 that $H_{\mathcal{N}}$ has a non-empty interior in $H$. Indeed, since $\Lambda_{\infty}$ is surjective it is open. The Kähler cone, $\mathcal{K} \subset H^{1,1}(X, \mathbb{R})$ is also open and $\Lambda_{\infty}(\mathcal{K})$ is contained in $H_{\mathcal{N}}$.

Theorem 3.2. ([Fav00, [Kis00]) Let $f: X \rightarrow X$ be a dominant meromorphic map and $T$ be a positive closed $(1,1)$ current on $X$. Then for every $x \in X \backslash I_{f}$

$$
\nu(T, f(x)) \leq \nu\left(f^{*} T, x\right) \leq C_{f} \nu(T, f(x))
$$

where $C_{f}>0$ is constant which does not depend on $T$.

Theorem 3.3. Let $f: X \rightarrow X$ be a dominant meromorphic map.

(1) For every $\alpha \in H_{\text {psef }}^{1,1}(X, \mathbb{R})$ and $p \in X \backslash I_{f}$

$$
\nu\left(f^{*} \alpha, p\right) \leq C_{f} \nu(\alpha, f(p))
$$

where $C_{f}$ is independent of $\alpha$. In particular, $f\left(E_{n n}\left(f^{*} \alpha\right) \backslash I_{f}\right) \subset E_{n n}(\alpha)$.

(2) Assume that $f$ is 1-regular and $\lambda:=\lambda_{1}(f)>1$. Then $E_{n n}(\alpha) \subset \mathcal{I}_{\infty}$ for every $\alpha \in H_{\mathcal{N}}$. In particular, $H_{\mathcal{N}} \subset \mathcal{E}_{1}$.

Proof. (1) We assume that $\alpha \in H_{\text {big }}^{1,1}(X, \mathbb{R})$. Let $p \in X \backslash I_{f}$ and $T_{\alpha}^{\text {min }} \in \alpha$ be a positive closed $(1,1)$ current with minimal singularities. Since $f^{*} T_{\alpha}^{\min } \in f^{*} \alpha$ by definition of $\nu\left(f^{*} \alpha, p\right)$ and by Theorem 3.2 we have

$$
\nu\left(f^{*} \alpha, p\right) \leq \nu\left(f^{*}\left(T_{\alpha}^{\min }\right), p\right) \leq C_{f} \nu\left(T_{\alpha}^{\min }, f(p)\right)=C_{f} \nu(\alpha, f(p))
$$

where the last equality follows from $\alpha \in H_{b i g}^{1,1}(X, \mathbb{R})$.

If $\alpha$ is merely psef then we consider $\alpha_{\delta}:=\alpha+\delta\{\omega\} \in H_{b i g}^{1,1}(X, \mathbb{R})$ for $\delta>0$. Now, by lower semi-continuity of $\nu(\cdot, p)$ and continuity of $f^{*}$ we get

$$
\nu\left(f^{*} \alpha, p\right) \leq \liminf _{\delta \rightarrow 0} \nu\left(f^{*} \alpha_{\delta}, p\right) .
$$

Since $\alpha_{\delta}$ is big, by above argument we also have

$$
\nu\left(f^{*} \alpha_{\delta}, p\right) \leq C_{f} \nu\left(\alpha_{\delta}, f(p)\right)
$$

for $\delta>0$. Then by sub-additivity and homogeneity of $\nu(\cdot, f(p))$ we get

$$
\nu\left(\alpha_{\delta}, f(p)\right) \leq \nu(\alpha, f(p))+\delta \nu(\{\omega\}, f(p))=\nu(\alpha, f(p))
$$

since $\{\omega\}$ is Kähler. Therefore, the assertion follows.

(2) Let $\alpha \in H_{\mathcal{N}}$ then $\alpha=\lim _{N \rightarrow \infty} \Lambda_{N} \beta$ for some $\beta \in H_{n e f}^{1,1}(X, \mathbb{R})$ and $f^{*} \alpha=\lambda \alpha$.

By part(1) we have

$$
\nu\left(\frac{1}{n^{m-1} \lambda^{n}}\left(f^{n}\right)^{*} \beta, p\right)=0
$$


for $p \notin I_{f^{n}}$. It follows from sub-additivity, lower semi-continuity of $\nu(\cdot, p)$ and continuity of $f^{*}$ that

$$
\nu(\alpha, p) \leq \liminf _{N \rightarrow \infty} \frac{1}{N} \sum_{n=1}^{N} \frac{1}{n^{m-1} \lambda^{n}} \nu\left(\left(f^{n}\right)^{*} \beta, p\right) .
$$

Thus, $\nu(\alpha, p)=0$ for $p \notin \mathcal{I}_{\infty}$.

Finaly, since $f$ is 1 -regular $\mathcal{I}_{\infty}$ does not contain any divisors hence, $\alpha \in \mathcal{E}_{1}$.

Without the assumption $\alpha \in H_{\mathcal{N}}$ the assertion of Theorem 3.3 (2) is not true in general. The following example was communicated by V. Guedj.

Example 3.4. Let $f: \mathbb{P}^{2} \rightarrow \mathbb{P}^{2}$ be a holomorphic map of degree $\lambda \geq 2$ with a totally invariant point p i.e. $f^{-1}(p)=p$. We define $\pi: X \rightarrow \mathbb{P}^{2}$ to be the blow up of $\mathbb{P}^{2}$ at $p$. Let $f_{X}$ denote the induced map and $E:=\pi^{-1}(p)$ denote the exceptional fiber. Then $f_{X}^{*}\{E\}=\lambda\{E\}$. Thus, $f_{X}^{*}: H^{1,1}(X, \mathbb{R}) \rightarrow H^{1,1}(X, \mathbb{R})$ is given by

$$
f_{X}^{*}=\left[\begin{array}{cc}
\lambda & 0 \\
0 & \lambda
\end{array}\right]
$$

Notice that the class $\{E\} \in H_{\text {psef }}^{1,1}(X, \mathbb{R})$ but $E \cdot E=-1$, hence, $\{E\} \notin H_{\mathcal{N}}=$ $H_{n e f}^{1,1}(X, \mathbb{R})$.

We also remark that in the above case $\lambda_{2}(f)=\lambda_{1}(f)^{2}$. It follows from DF01 that if $\operatorname{dim}_{\mathbb{C}}(X)=2$ and $r_{1}(f)^{2}>\lambda_{2}(f)$ then $r_{1}(f)$ is a simple root of the characteristic polynomial of $f^{*}$.

The following follows from Proposition 2.9.

Corollary 3.5. Let $f$ and $\alpha \in H_{\mathcal{N}}$ be as in Theorem 3.3 (2). Then every $\alpha$-negative curve $C \subset \mathcal{I}_{\infty}$.

Corollary 3.6. Let $f: X \rightarrow X$ be a dominant meromorphic map such that $\operatorname{dim}\left(I_{f}\right)=0$ then $f^{*}\left(H_{n e f}^{1,1}(X, \mathbb{R})\right) \subset H_{n e f}^{1,1}(X, \mathbb{R})$. In particular, if $X$ is a compact Kähler surface $f^{*}$ preserves the nef cone.

Proof. Let $\alpha \in H_{n e f}^{1,1}(X, \mathbb{R})$ then it follows from Theorem 3.3 that $E_{n n}\left(f^{*} \alpha\right) \subset I_{f}$ and since $\operatorname{dim}\left(I_{f}\right)=0, E_{n n}(\alpha)$ is a finite set. Thus, the assetion follows from the regularization argument of [Dem92, Lemma 6.3].

If $X$ is a compact Kähler surface then the cone $\mathcal{E}_{1}$ coincides with $H_{n e f}^{1,1}(X, \mathbb{R})$ ([Bou04]). Thus, $f^{*}\left(\mathcal{E}_{1}\right) \subset \mathcal{E}_{1}$ when $\operatorname{dim}_{\mathbb{C}} X=2$.

However, if $\operatorname{dim}_{\mathbb{C}} X \geq 3$ this is no longer true as the following example shows:

Example: Let

$$
\begin{gathered}
J: \mathbb{P}^{3} \rightarrow \mathbb{P}^{3} \\
J\left[x_{0}: x_{1}: x_{2}: x_{3}\right]=\left[x_{1} x_{2} x_{3}: x_{0} x_{2} x_{3}: x_{0} x_{1} x_{3}: x_{0} x_{1} x_{2}\right]
\end{gathered}
$$

and $L \in \operatorname{Aut}\left(\mathbb{P}^{3}\right)$ given by the matrix

$$
L=\left[\begin{array}{llll}
1 & 0 & 0 & 1 \\
0 & 0 & 1 & 1 \\
0 & 1 & 0 & 1 \\
0 & 1 & 1 & 0
\end{array}\right]
$$


We consider the birational map $f=L \circ J: \mathbb{P}^{k} \rightarrow \mathbb{P}^{k}$

$f\left[x_{0}: x_{1}: x_{2}: x_{3}\right]=\left[\left(x_{0}+x_{3}\right) x_{1} x_{2}:\left(x_{2}+x_{3}\right) x_{0} x_{1}:\left(x_{1}+x_{3}\right) x_{0} x_{2}:\left(x_{1}+x_{2}\right) x_{0} x_{3}\right]$

Notice that $f$ has four exceptional hypersurfaces: $\left\{\Sigma_{0}, \Sigma_{1}, \Sigma_{2}, \Sigma_{3}\right\}$ where $\Sigma_{i}:=$ $\left\{x_{i}=0\right\}$ with the following orbit data:

$$
\begin{aligned}
\Sigma_{0} \rightarrow e_{0} & :=[1: 0: 0: 0] \rightsquigarrow \Sigma_{\beta} \\
\Sigma_{1} \rightarrow e_{23} & :=[0: 0: 1: 1] \rightsquigarrow l_{1} \rightarrow e_{23} \\
\Sigma_{2} \rightarrow e_{13} & :=[0: 1: 0: 1] \rightsquigarrow l_{2} \rightarrow e_{13} \\
\Sigma_{3} & \rightarrow[1: 1: 1: 0] \circlearrowleft
\end{aligned}
$$

where " $\rightsquigarrow "$ indicates the total transform under $f$ and $\Sigma_{\beta}=\left\{\left[x_{0}: x_{1}: x_{2}: x_{3}\right] \in\right.$ $\left.\mathbb{P}^{3}: 2 x_{0}-x_{1}-x_{2}+x_{3}=0\right\}, l_{1} \subset \Sigma_{1}$ is the line passing through $e_{0}$ and $e_{23}$ and $l_{2} \subset \Sigma_{2}$ is the line passing through $e_{0}$ and $e_{13}$.

We define the complex manifold $X$ to be $\mathbb{P}^{3}$ blown up at $e_{0}, e_{23}$ and $e_{13}$ successively. We denote the exceptional fibers on $e_{0}, e_{23}$ and $e_{1,3}$ by $E_{0}, E_{23}$ and $E_{13}$ respectively and the induced map by $f_{X}: X \rightarrow X$. Then we have the following orbit data

$$
\begin{gathered}
\Sigma_{0} \rightarrow E_{0} \rightarrow \Sigma_{\beta} \\
\Sigma_{1} \rightarrow E_{23} \rightarrow l_{1} \rightarrow \gamma \rightarrow l_{1} \\
\Sigma_{2} \rightarrow E_{13} \rightarrow l_{2} \rightarrow \sigma \rightarrow l_{2} \\
\Sigma_{3} \rightarrow[1: 1: 1: 0] \circlearrowleft
\end{gathered}
$$

where $\gamma \subset E_{23}$ and $\sigma \subset E_{13}$ are lines which are regular. It follows that no exceptional hypersurface of $f_{X}$ is mapped into indeterminacy locus $I_{f_{X}}$, thus $f_{X}$ is 1-regular. Now, $\left\langle H_{X}, E_{0}, E_{23}, E_{13}\right\rangle$ forms a basis for $H^{1,1}(X)$ and the the action $f_{X}^{*}: H^{1,1}(X) \rightarrow H^{1,1}(X)$ with respect to this ordered basis is given by the integer coefficient matrix

$$
f_{X}^{*}=\left[\begin{array}{cccc}
3 & 1 & 1 & 1 \\
-2 & 0 & -1 & -1 \\
-1 & -1 & -1 & 0 \\
-1 & -1 & 0 & -1
\end{array}\right]
$$

the charactersitic polynomial of $f_{X}^{*}$ is $\chi(x)=x^{4}-x^{3}-3 x^{2}+x+2$ and the first dynamical degree of $f$, the largest root of $\chi(x), \lambda_{1}(f)=2$ is a simple eigenvalue.

Let $\widetilde{\Sigma_{1}} \subset X$ denote the strict transform of $\Sigma_{1}$. Since $e_{0}, e_{23} \in \Sigma_{1} \subset \mathbb{P}^{3}$, the class $\alpha:=\left\{\widetilde{\Sigma_{1}}\right\}=H_{X}-E_{0}-E_{23}$. Moreover, $\left\{\widetilde{\Sigma_{1}}\right\}$ is nef in codimension 1. Indeed, for any hyperplane $H \subset \mathbb{P}^{3}$ containing $e_{0}$ and $e_{23}$ which does not contain $e_{13}, \widetilde{H}$ cohomologous to $\widetilde{\Sigma_{1}}$ but this is a 1-parameter family of hyperplanes and $[\widetilde{H}] \in \alpha$ defines a positive closed $(1,1)$ current. Thus, we infer that $\nu\left(T_{\alpha}^{\min }, x\right)=0$ for every $x \notin l_{1}$. Hence, by Proposition $2.4 E_{n n}\left(\left\{\widetilde{\Sigma_{1}}\right\}\right) \subset l_{1}$. Now, since $\widetilde{\Sigma_{1}} \cdot l_{1}=-1$ by Proposition $2.9 l_{1} \subset E_{n n}$. Therefore, $E_{n n}\left(\left\{\widetilde{\Sigma_{1}}\right\}\right)=l_{1}$. On the other hand,

$$
f^{*}(\alpha)=f_{X}^{*}\left(H_{X}-E_{0}-E_{23}\right)=H_{X}-E_{0}+E_{23}=\left\{\widetilde{H}_{0}\right\}+E_{23}
$$

where $H_{0}$ is any hyperplane in $\mathbb{P}^{3}$ containing $e_{0}$ which does not contain $e_{23}$ nor $e_{13}$. Since this is a 2-parameter family by the same argument above we see that $E_{n n}\left(H_{X}-E_{0}+E_{23}\right) \subset E_{23}$. Moreover, for a generic line $\sigma \subset E_{23}$ we have $E_{23} \cdot \sigma=-1$. Therefore, $E_{n n}\left(H_{X}-E_{0}+E_{23}\right)=E_{23}$ hence $f^{*}(\alpha) \notin \mathcal{E}_{1}$. 


\section{Green Currents}

Proof of Theorem 1.2. We fix a smooth representative $\theta \in \alpha$ and let

$$
T_{\alpha}^{\text {min }}=\theta+d d^{c} v_{\alpha}^{\text {min }}
$$

denote the current of minimal singularities. Since $\alpha$ is invariant by $d d^{c}$-lemma GH78, p 149], we can write

$$
\frac{1}{\lambda} f^{*} T_{\alpha}^{m i n}=\theta+d d^{c} \phi_{1}
$$

where $\phi_{1}$ is a qpsh function thus, we can assume that $\phi_{1} \leq 0$. Then by definition of $v_{\alpha}^{\min }$ we have $\phi_{1} \leq v_{\alpha}^{\min }$.

Now, by using invariance of $\alpha$ again we write

$$
\frac{1}{\lambda^{2}}\left(f^{2}\right)^{*} T_{\alpha}^{m i n}=\theta+d d^{c} \phi_{2}
$$

since $f$ is 1 -regular by (4.2) we obtain

$$
\frac{1}{\lambda^{2}}\left(f^{2}\right)^{*} T_{\alpha}^{m i n}=\frac{1}{\lambda} f^{*}\left(\frac{1}{\lambda} f^{*} T_{\alpha}^{m i n}\right)=\frac{1}{\lambda} f^{*}(\theta)+\frac{1}{\lambda} d d^{c} \phi_{1} \circ f
$$

and using (4.1) and (4.2) we have

$$
\frac{1}{\lambda} f^{*}(\theta)=\theta+d d^{c}\left(\phi_{1}-\frac{1}{\lambda} v_{\alpha}^{\min } \circ f\right)
$$

and substituting (4.5) in (4.4) we obtain

$$
\frac{1}{\lambda^{2}}\left(f^{2}\right)^{*} T_{\alpha}^{m i n}=\theta+d d^{c}\left(\phi_{1}+\frac{1}{\lambda}\left(\phi_{1}-v_{\alpha}^{m i n}\right) \circ f\right)
$$

therefore, by adding a constant if necessary we can choose

$$
\phi_{2}=\phi_{1}+\frac{1}{\lambda}\left(\phi_{1}-v_{\alpha}^{m i n}\right) \circ f
$$

since $\phi_{1} \leq v_{\alpha}^{\min }$ we get $\phi_{2} \leq \phi_{1}$.

Iterating this argument we obtain

$$
\frac{1}{\lambda^{n}}\left(f^{n}\right)^{*} T_{\alpha}^{\min }=\theta+d d^{c} \phi_{n}
$$

where

$$
\phi_{n}=\phi_{1}+\sum_{j=1}^{n-1} \frac{1}{\lambda^{j}}\left(\phi_{1}-v_{\alpha}^{\text {min }}\right) \circ f^{j}
$$

for $n \geq 2$ and $\left\{\phi_{n}\right\}$ is a decreasing sequence of negative qpsh functions. Thus, by Hartogs lemma either $\phi_{n}$ converges uniformly to $-\infty$ or $\phi_{n}$ converges to some qpsh function $g$. We will show that the former case is not possible by using a trick due to Sibony Sib99:

Let $R \in \alpha$ be a positive closed $(1,1)$ current. We consider the Cesaro means of the form

$$
R_{N}=\frac{1}{N} \sum_{i=0}^{N-1} \frac{1}{\lambda^{i}}\left(f^{i}\right)^{*} R
$$

notice that $R_{N}$ 's are positive closed $(1,1)$ currents and $\left\|R_{N}\right\|=\|R\|$ where $\|R\|=$ $\int_{X} R \wedge w^{k-1}$. Therefore we can extract a subsequence $R_{N_{k}} \rightarrow S$ for some positive closed current $S \in \alpha$ such that $f^{*} S=\lambda S$ and we have

$$
S=\theta+d d^{c} u
$$


where $u$ is a qpsh function. Then, by invariance of $\mathrm{S}$ we get

$$
\frac{1}{\lambda}\left(f^{*} \theta+d d^{c} u \circ f\right)=\theta+d d^{c} u
$$

and by (4.5) we infer

$$
\theta+d d^{c}\left(\phi_{1}-\frac{1}{\lambda}\left(v_{\alpha}^{\min }+u\right) \circ f\right)=\theta+d d^{c} u .
$$

Thus, by adding a constant to $u$ we can assume that

$$
\phi_{1}-\frac{1}{\lambda} v_{\alpha}^{\min } \circ f=u-\frac{1}{\lambda} u \circ f
$$

Pulling back (4.7) by $\frac{1}{\lambda} f$ and adding the result to (4.7) again we obtain

$$
\phi_{n}-\frac{1}{\lambda^{n}} v_{\alpha}^{\min } \circ f^{n}=u-\frac{1}{\lambda^{n}} u \circ f^{n}
$$

Since $u$ is qpsh it's bounded from above $u \leq C$ and we have,

$$
\phi_{n} \geq u+\frac{1}{\lambda^{n}} v_{\alpha}^{\min } \circ f^{n}-\frac{1}{\lambda^{n}} C .
$$

Thus, we infer that $\phi_{n}$ converges to $g_{\theta}$ for some qpsh function $g_{\theta}$. We denote the limit current by $T_{\alpha}=\theta+d d^{c} g_{\theta}$. Since it's a limit of positive closed currents belonging to $\alpha, T_{\alpha} \in \alpha$ is a positive closed current. Moreover, by continuity of $f^{*}$ we have $f^{*} T_{\alpha}=\lambda T_{\alpha}$. Now, we will show that $g_{\theta}$ depends only on the class $\alpha$ :

First of all, since $\frac{1}{\lambda^{n}} v_{\alpha}^{\text {min }} \circ f^{n} \rightarrow 0$ in $L^{1}(X)$ we get

$$
T_{\alpha}=\lim _{n \rightarrow \infty} \frac{1}{\lambda^{n}}\left(f^{n}\right)^{*} T_{\alpha}^{m i n}=\lim _{n \rightarrow \infty} \frac{1}{\lambda^{n}}\left(f^{n}\right)^{*} \theta
$$

Now, if $\theta^{\prime}$ is another smooth form representing the class $\alpha$ then by $d d^{c}$-lemma we can write $\theta^{\prime}=\theta+d d^{c} \varphi$ where $\varphi$ is a smooth function and since $\mathrm{X}$ is compact $\varphi$ is bounded. Therefore, the current $T_{\alpha}=\theta+d d^{c} g_{\alpha}$ is independent of the choice of the representative form. So far, we have proved the first part.

To prove (1): let $\sigma \in \alpha$ be an invariant current i.e. $f^{*} \sigma=\lambda \sigma$ and $\sigma=\theta+d d^{c} \psi$ for some qpsh function $\psi \leq 0$. Then by the same argument as above we obtain

$$
\phi_{n}-\frac{1}{\lambda^{n}} v_{\alpha}^{\min } \circ f^{n}=\psi-\frac{1}{\lambda^{n}} \psi \circ f^{n}+C \sum_{j=0}^{n-1} \frac{1}{\lambda^{j}}
$$

thus, $g_{\alpha}+C_{1} \geq \psi$.

Proof of (2) appears in the literature, see Sib99, Gue04.

Remark 4.1. Notice that in the proof of Theorem 4.1 to get the convergence $\lim _{n \rightarrow \infty} \frac{1}{\lambda^{n}}\left(f^{n}\right)^{*} T_{\alpha}^{\text {min }}=T_{\alpha}$ we only need that $\left\{\frac{1}{\lambda^{n}} v_{\alpha}^{\text {min }} \circ f^{n}\right\}$ is locally bounded near some point $x \in X$.

Theorem 1.2 was proved by Fornaess and Sibony when $X=\mathbb{P}^{k}$ (see [FS95] and Sib99]). More recently, it was proved in Gue04 under a cohomological assumption which we replace here by a weaker dynamical assumption. See also [FG01, [DF01, Gue02, DG09, DDG10 for similar constructions and Ngu for the case of non 1-regular meromorphic self maps of $\mathbb{P}^{k}$.

It seems that the first two assumptions (1-regularity and $\lambda_{1}>1$ ) in Theorem 1.2 are quite natural. Indeed, if $\lambda_{1}(f)=1$ then it follows from concavity of the 
function $j \rightarrow \log \left(\lambda_{j}\right)$ and the upper bound for the entropy [DS04] that $h_{\text {top }}(f)=0$.

What about the condition $(\star)$ ? If $\alpha$ is a Kähler class then $v_{\alpha}^{\min } \equiv 0$ thus, $(\star)$ holds. More generally, if $\alpha$ can be represented by a semi-positive form then $v_{\alpha}^{\text {min }}$ is bounded hence $(\star)$ holds. This is the case for complex homogenous manifolds (i.e. when the group of automorphisms Aut(X) acts transitively on X). Indeed, if $\mathrm{X}$ is a complex homogeneous manifold, then every positive closed current $\mathrm{T}$ can be approximated by positive smooth forms $\theta_{\epsilon} \in\{T\}$ (see Huc94). Therefore any psef class can be represented by a semi-positive form. If $X$ is a compact Kähler surface and $\lambda_{1}(f)^{2}>\lambda_{2}(f)$, it is well known that $\lambda_{1}$ is a simple eigenvalue (see [DF01]). Furthermore, the corresponding eigenvector $\alpha \in H_{\text {psef }}^{1,1}(X, \mathbb{R})$ can be represented by a positive closed $(1,1)$ current with bounded potentials (see DDG10]). Thus, in this case $(\star)$ holds.

However, there are some examples for which the condition $(\star)$ does not hold:

Example 4.2. Let $f: \mathbb{P}^{2} \rightarrow \mathbb{P}^{2}$

$$
f\left[x_{0}: x_{1}: x_{2}\right]=\left[x_{0}^{2}: x_{1}^{2}: x_{2}^{2}\right] .
$$

Then $f$ is a holomorphic map with the totally invariant point $p=[1: 0: 0]$ i.e. $f^{-1}(p)=p$. Let $\pi: X \rightarrow \mathbb{P}^{2}$ denote the blow-up of $\mathbb{P}^{2}$ at $p$. We let $f_{X}$ denote the induced map and $E:=\pi^{-1}(p)$ denote the exceptional fiber. Then $f_{X}^{*}\{[E]\}=$ $2\{[E]\}$ where $[E]$ denotes the current of integration along $E$. Notice that $\alpha:=\{[E]\}$ contains only one positive closed $(1,1)$ current namely, $[E]$. Thus, $T_{\alpha}^{\text {min }}=[E]$ and $E=\left\{v_{\alpha}^{\text {min }}=-\infty\right\}$. We will show that

$$
\operatorname{Vol}\left(\frac{1}{2^{n}} v_{\alpha}^{\min } \circ f_{X}^{n}<-1\right) \not \rightarrow 0 .
$$

Indeed, we choose the local coordinates $(s, \eta)$ on $X$ such that $\pi(s, \eta)=[1: s: s \eta]$ Then in these coordinates $E=\{s=0\}, v_{\alpha}^{\min }(s, \eta)=\log |s|$ and

$$
\frac{1}{2^{n}} v_{\alpha}^{\min } \circ f_{X}^{n}=\log |s|
$$

thus,

$$
\left\{|s|<e^{-1}\right\} \subset\left\{\frac{1}{2^{n}} v_{\alpha}^{\text {min }} \circ f_{X}^{n}<-1\right\}
$$

for every $n \in \mathbb{N}$ and the claim follows.

Proposition 4.3. Let $f: X \rightarrow X$ be a dominant 1-regular meromorphic map and $f^{*} \alpha=\lambda \alpha$ for some $\alpha \in H_{\text {psef }}^{1,1}(X, \mathbb{R})$ with $\lambda>1$. Assume that there exists $a$ positive closed $(1,1)$ current $T:=\theta+d d^{c} \phi$ such that

$$
T=\lim _{n \rightarrow \infty} \frac{1}{\lambda^{n}}\left(f^{n}\right)^{*} \theta
$$

for some (equivalently for every) smooth form $\theta \in \alpha$. Then $(\star)$ holds.

Proof. Since $\theta \in \alpha$ is a smooth form we have $T=\theta+d d^{c} \phi$ where $\phi$ is a qpsh function and $\phi \leq v_{\alpha}^{\text {min }}+O(1)$. It's enough to show that $\frac{1}{\lambda^{n}} \phi \circ f^{n} \rightarrow 0$ in $L^{1}(X)$. Let

$$
\frac{1}{\lambda} f^{*} \theta=\theta+d d^{c} \gamma
$$

for some $\gamma \in L^{1}(X)$. By continuity of $f^{*}$ we have $f^{*} T=\lambda T$. Thus, by adding a constant to $\gamma$ if necessary we may assume that

$$
\gamma+\frac{1}{\lambda} \phi \circ f=\phi
$$


then we have

$$
\phi=\sum_{i=0}^{n-1} \frac{1}{\lambda^{i}} \gamma \circ f^{i}+\frac{1}{\lambda^{n}} \phi \circ f^{n}
$$

and by assumption $\sum_{i=0}^{n-1} \frac{1}{\lambda^{2}} \gamma \circ f^{i} \rightarrow \phi$ in $L^{1}(X)$ hence, $\frac{1}{\lambda^{n}} \phi \circ f^{n} \rightarrow 0$ in $L^{1}(X)$.

Thus, it follows from the following theorem that if $\lambda_{1}(f)>1$ is simple and $\alpha$ is nef then condition $(\star)$ holds.

Theorem 4.4. DG09 Let $f: X \rightarrow X$ be a dominant meromorphic map. Assume that $f$ is 1-regular and $\lambda:=\lambda_{1}(f)>1$ is the unique simple eigenvalue and $\alpha \in$ $H^{1,1}(X, \mathbb{R})$ is the corresponding eigenvector. If $\alpha$ is nef then for every smooth form $\theta \in \alpha$ we have the limit $T_{\alpha}:=\lim _{n \rightarrow \infty} \frac{1}{\lambda^{n}}\left(f^{n}\right)^{*}(\theta)$ which depends only on the class $\alpha$. Moreover $T_{\alpha}$ is a positive closed $(1,1)$ current satisfying $f^{*} T_{\alpha}=\lambda T_{\alpha}$.

\section{An Algebraic Criterion}

The following result is a consequence Hodge index theorem [KM98, Lemma 3.39]:

Lemma 5.1 (Negativity Lemma). Let $\pi: Z \rightarrow Y$ be a proper birational morphism between normal projective varieties $Z$ and $Y$. Let $-E$ be a $\pi$-nef $\mathbb{R}$-divisor on $Z$ (that is $(-E) \cdot C \geq 0$ for every $\pi$-exceptional curve $C$ ). Then

(1) $E$ is effective if and only if $\pi_{*} E$ is effective

(2) Assume that $E$ is effective. Then for every $y \in Y$ either $\pi^{-1}(y) \subset E$ or $\operatorname{supp}(E) \cap \pi^{-1}(y)=\emptyset$.

Proposition 5.2. Let $X$ be a projective manifold and $f: X \rightarrow X$ be a dominant rational map and $\omega$ be a Kähler form. If $p \in I_{f}$ then $\nu\left(f^{*} \omega, p\right)>0$.

Proof. We consider the pull-backs

$$
\left(\pi_{1}\right)^{*} f^{*} \omega=\left(\pi_{1}\right)^{*}\left(\pi_{1}\right)_{*}\left(\pi_{2}\right)^{*} \omega=\left(\pi_{2}\right)^{*} \omega+E
$$

where $E$ is a (possibly trivial) $\pi_{1}$-exceptional divisor. We claim that $E$ is a nontrivial effective divisor. Indeed, for any $\pi_{1}$-exceptional curve $C$ we have

$$
\left(\pi_{1}\right)^{*} f^{*} \omega \cdot C=0
$$

and since $\omega$ is a Kähler form we get

$$
0 \leq\left\langle\omega, \pi_{2}(C)\right\rangle=(-E) \cdot C
$$

Thus, by Negativity lemma we conclude that $E$ is effective.

Let us fix $p \in I_{f}$. Since $\operatorname{dim}(f(p)) \geq 1$, there exists a curve $C \subset \pi_{1}^{-1}(p)$ such that $C \not \subset \mathcal{E}\left(\pi_{2}\right)$ and by above argument $E \cdot C<0$ hence $E$ is non-trivial. Moreover, $\pi_{1}^{-1}(p) \subset E$.

Since the left hand side of (5.1) defines a positive closed $(1,1)$ current we infer that $\nu\left(\pi_{1}^{*} f^{*} \omega, q\right)=\operatorname{mult}_{q}(E)$ for any $q \in \pi_{1}^{-1}(p)$ and

$$
0<\nu\left(\left(\pi_{1}\right)^{*} f^{*} \omega, q\right)
$$

Now, it follows from Theorem 3.2 that

$$
0<\nu\left(\left(\pi_{1}\right)^{*} f^{*} \omega, q\right) \leq C \nu\left(f^{*} \omega, p\right)
$$

where $C>0$ is a constant which does not depend on $f^{*} \omega$. 
The following result is well-known when $X=\mathbb{P}^{k}$ [FS95] or $X$ is a compact Kähler surface [DF01]. To our knowledge it is new in this generality:

Theorem 5.3. Let $X$ be a projective manifold and $f: X \rightarrow X$ be a dominant rational map. Then the following are equivalent:

(i) $\left(f^{n}\right)^{*} T=\left(f^{*}\right)^{n} T$ for every $T \in \mathcal{T}(X)$ and $n=1,2, \ldots$

(ii) $\left(f^{*}\right)^{n} \omega=\left(f^{n}\right)^{*} \omega$ for every Kähler form $\omega$ on $X$ and $n=1,2, \ldots$

(iii) $f$ is 1-regular

(iv) There is no exceptional hypersurface $H$ and $n \in \mathbb{N}$ such that $f^{n}(H-$ $\left.I_{f^{n}}\right) \subset I_{f}$.

Proof. $(i) \Longrightarrow(i i) \Longrightarrow($ iii $)$ is clear.

(iii) $\Longrightarrow($ iv $)$ Let be $\omega$ be a Kähler form. Assume that there exists an exceptional hypersurface $H$ such that $f^{n}\left(H-I_{f}\right) \subset I_{f}$ for some $n$. By replacing $f$ with $f^{n}$ we may assume that $f\left(H-I_{f}\right) \subset I_{f}$. Then by Proposition 5.2 for each $p \in f\left(V-I_{f}\right) \subset$ $I_{f}$ we have $\nu\left(f^{*} \omega, p\right)>0$ but this implies that $\nu\left(\left(f^{*}\right)^{2} \omega, q\right)>0$ for every $q \in H-I_{f}$. However, $\left(f^{2}\right)^{*} \omega$ is an $L_{l o c}^{1}$ coefficient form and does not charge $H$ and hence the cohomology classes of $\left(f^{*}\right)^{2} \omega$ and $\left(f^{2}\right)^{*} \omega$ are different.

(iv) $\Longrightarrow(i)$ Let $T \in \mathcal{T}(X)$. Notice that $f^{n-1}$ and $f^{n}$ are both holomorphic on $\left.X-\left(I_{f} \cup f^{-1}\left(I_{f}\right)\right) \cdots \cup f^{n-1}\left(I_{f}\right)\right)$ Thus, $\left(f^{n}\right)^{*} T=\left(f^{*}\right)\left(f^{n-1}\right)^{*} T$ on this set. Since there is no hypersurface contained in $\left.X-\left(I_{f} \cup f^{-1}\left(I_{f}\right)\right) \cdots \cup f^{n-1}\left(I_{f}\right)\right)$ we get the equality on $X$.

Lemma 5.4. Let $\pi: Z \rightarrow Y$ be a proper modification between smooth projective varieties. Let $\eta$ be a smooth closed real $(1,1)$ form on $Z$ such that $\langle\eta, C\rangle \geq 0$ for every $\pi$-exceptional curve $C$. Then $\pi_{*} \eta$ has potentials bounded from above.

Proof. Let $\pi_{*} \eta=\eta^{\prime}+d d^{c} u$ for some smooth form $\eta^{\prime}$ and $u \in L^{1}(X)$. We claim that $u$ is bounded from above. Indeed,

$$
\pi^{*} \pi_{*} \eta=\pi^{*} \eta^{\prime}+d d^{c}(u \circ \pi)=\eta+E
$$

where $E$ is an $\mathbb{R}$ divisor supported in $\mathcal{E}(\pi)$. Since

$$
0 \leq\langle\eta, C\rangle=\langle-E, C\rangle
$$

for every $\pi$-exceptional curve $C$ by negativity lemma $E$ is an effective divisor. Hence, $u \circ \pi$ is qpsh on $Z$ and bounded from above. Thus, so is $u$.

Proposition 5.5. Let $X$ be a projective manifold and $f: X \rightarrow X$ be a dominant rational map. Let $\theta$ be a smooth closed real $(1,1)$ form on $X$ such that $\langle\theta, C\rangle \geq 0$ for every curve $C \subset E_{f}^{-}:=f\left(I_{f}\right)$ then the potentials of $f^{*} \theta$ are bounded from above.

Proof. We write $f^{*} \theta=\left(\pi_{1}\right)_{*}\left(\pi_{2}\right)^{*} \theta$ where $\left(\pi_{2}\right)^{*} \theta$ is a smooth form on the desingularization of the graph of $f, \tilde{\Gamma} \subset X \times X$. Notice that for any $\pi_{1}$-exceptional curve $C \subset \tilde{\Gamma}, \pi_{2}(C)$ is either a point in $X$ or a curve in $E_{f}^{-}$. Thus, we have

$$
\left\langle\pi_{2}^{*} \theta, C\right\rangle=\left\langle\theta, \pi_{2}(C)\right\rangle \geq 0
$$

Then, applying Lemma 5.4 with $\eta=\left(\pi_{2}\right)^{*} \theta$ the assertion follows.

For a convex cone $\mathcal{C}$ in a finite dimensional vector space $V$ we define the dual cone $\mathcal{C}^{\vee}$ to be the set of linear forms in $V^{*}$ which have non-negative values on $\mathcal{C}$. Moreover, by Hahn-Banach thoerem we have $\mathcal{C}^{\vee \vee}=\overline{\mathcal{C}}$. 
Theorem 5.6. Let $X$ be a projective manifold and $f: X \rightarrow X$ be a 1-regular dominant rational map. We assume that $\lambda_{1}(f)>1$ is a simple eigenvalue of $f^{*}$ and $\alpha$ denote the corresponding eigenvector. If $\alpha \cdot C \geq 0$ for every curve $C \subset E_{f}^{-}$ then for every smooth representative $\theta \in \alpha$ we have

$$
T_{\alpha}=\lim _{n \rightarrow \infty} \frac{1}{\lambda^{n}}\left(f^{n}\right)^{*} \theta
$$

exists. Moreover, $T_{\alpha}$ is a positive closed $(1,1)$ current such that $f^{*} T_{\alpha}=\lambda T_{\alpha}$.

Proof. We will sketch the proof: Let $\theta \in \alpha$ be a smooth representative then by $d d^{c}$-lemma we have

$$
\frac{1}{\lambda} f^{*} \theta=\theta+d d^{c} \gamma
$$

and by Proposition 5.5 $\gamma \in L^{1}(X)$ is bounded from above. Thus, we may assume that $\gamma \leq 0$. Iterating this equation we get

$$
\frac{1}{\lambda^{n}}\left(f^{n}\right)^{*} \theta=\theta+d d^{c} \gamma_{n}
$$

where

$$
\gamma_{n}=\sum_{j=0}^{n-1} \frac{1}{\lambda^{j}} \gamma \circ f^{j} .
$$

Then $\left\{\gamma_{n}\right\}$ is a decreasing sequence in $L^{1}(X)$. It follows from Sibony's argument Sib99] that $\left\{\gamma_{n}\right\} \geq \phi$ for some qpsh function $\phi$. Thus, $\gamma_{n} \rightarrow \gamma_{\infty}$ for some $\gamma_{\infty} \in$ $L^{1}(X)$. Therefore,

$$
T_{\alpha}:=\theta+d d^{c} \gamma_{\infty}
$$

defines a closed $(1,1)$ current. It follows from continuity of $f^{*}$ that $f^{*} T_{\alpha}=\lambda T_{\alpha}$.

It remains to show that $T_{\alpha}$ is positive. We will follow the arguments from BS92] and DG09. It is enough to show that for every smooth cutoff function $\chi$ supported in a coordinate chart $U \subset X$ and positive $(k-1, k-1)$ form $\sigma$ which is constant relative the coordinates on $U$

$$
\langle T, \chi \sigma\rangle \geq 0 \text {. }
$$

Since $\lambda$ is simple it follows form [BS92], Lemma 1.3] that the sequence $\left\{\frac{1}{\lambda^{n}}\left(f^{n}\right)_{*}(\chi \sigma)\right\}$ has weak limit points which are positive and closed. Moreover, since $\left.\left(f_{*}\right)\right|_{H^{k-1, k-1}(X, \mathbb{R})}$ preserves classes; these limit points belongs to the dual of the psef cone. Thus,

$$
\left\langle T_{\alpha}, \chi \sigma\right\rangle=\lim _{n_{k} \rightarrow \infty}\left\langle\frac{1}{\lambda^{n_{k}}}\left(f^{n_{k}}\right)^{*} \theta, \chi \sigma\right\rangle=\langle\theta, S\rangle \geq 0
$$

where $S=\lim _{n_{k} \rightarrow \infty} \frac{1}{\lambda^{n_{k}}}\left(f^{n_{k}}\right)_{*}(\chi \sigma)$.

Note that if $\alpha$ is nef then $\alpha \cdot C \geq 0$ for every curve $C$. This is the case when $X$ is a compact Kähler surface and $\lambda_{1}(f)>\lambda_{2}(f)$ and the corresponding results were obtained in DDG10] as a consequence of so called "push-pull formula" (DF01]).

If there exists an irreducible curve $C \subset E_{f}^{-}$such that $\alpha \cdot C<0$ then $C \subset E_{n n}(\alpha)$. Thus, if $\operatorname{dim}\left(E_{n n}(\alpha) \cap E_{f}^{-}\right)=0$ then $\alpha \cdot C \geq 0$ for every curve $C \subset E_{f}^{-}$.

The following result follows from Proposition 4.3

Corollary 5.7. Let $f: X \rightarrow X$ and $\alpha$ be as in Theorem 5.6 then

$$
\frac{1}{\lambda^{n}} v_{\alpha}^{\text {min }} \circ f^{n} \rightarrow 0 \text { in } L^{1}(X) \text {. }
$$




\section{Noetherian Mappings}

Let $\mathbb{P}^{d}$ denote the complex projective space of dimension $d$ and for a point $x \in \mathbb{P}^{d}$

$$
x=\left[x_{0}: x_{1}: \cdots: x_{d}\right]
$$

denotes the homogenous coordinates on $\mathbb{P}^{d}$. For a subset $I \subset\{0,1, . ., d\}$ we denote its complement by $\hat{I}:=\{0,1, . ., d\}-I$ and its cardinality by $|I|$. We also define the sets

$$
\mathcal{D}_{I}:=\left\{\left[x_{0}: \cdots: x_{d}\right] \in \mathbb{P}^{d}: x_{i_{1}}=x_{i_{2}} \text { for every } i_{1}, i_{2} \in \hat{I}\right\} .
$$

In particular, if $I=\{i\}$ then we set $\mathcal{D}_{i}:=\mathcal{D}_{\{i\}}$ which is a complex line. We also denote

$$
\Sigma_{I}:=\left\{\left[x_{0}: \cdots: x_{d}\right] \in \mathbb{P}^{d}: x_{i}=0 \text { for } i \in I\right\} .
$$

In this section, we consider the maps of the form $f=L \circ J: \mathbb{P}^{d} \rightarrow \mathbb{P}^{d}$ where $J: \mathbb{P}^{d} \rightarrow \mathbb{P}^{d}$ is the involution defined by

$$
J\left[x_{0}: x_{1}: \cdots: x_{d}\right]=\left[x_{0}^{-1}: x_{1}^{-1}: \cdots: x_{d}^{-1}\right]=\left[x_{\hat{0}}: x_{\hat{1}}: \cdots: x_{\hat{d}}\right]
$$

with $x_{\hat{\jmath}}:=\prod_{\substack{i=1 \\ i \neq j}}^{d} x_{i}$ and $L$ is a linear map given by $(d+1) \times(d+1)$ matrix of the form

$$
L=\left[\begin{array}{ccccc}
a_{0}-1 & a_{1} & a_{2} & \ldots & a_{d} \\
a_{0} & a_{1}-1 & a_{2} & \ldots & a_{d} \\
a_{0} & a_{1} & a_{2}-1 & \ldots & a_{d} \\
\vdots & \vdots & \vdots & \ddots & \vdots \\
a_{0} & a_{1} & a_{2} & \ldots & a_{d}-1
\end{array}\right]
$$

with $a_{j} \in \mathbb{C}$ and $\sum_{j=0}^{d} a_{j}=2$. It follows that $\operatorname{det}(L)=(-1)^{d}$ and $L$ is involutive that is $L=L^{-1}$ in $P G L(d+1, \mathbb{C})$. A map of this form is called Noetherian mapping in BHM03. Notice that $f$ is a birational mapping with $f^{-1}=J \circ L$. Moreover, the indeterminacy locus is given by

$$
I_{f}=\bigcup_{|I| \geq 2} \Sigma_{I}
$$

For a point $p \in \mathbb{P}^{d}$, we define it's orbit $\mathcal{O}(p)$ as follows: $\mathcal{O}(p)=\{p\}$ if $p \in I_{f}$ and $\mathcal{O}(p)=\left\{p, f(p), f^{2}(p), . ., f^{N-1}(p)\right\}$ if $f^{j}(p) \notin I_{f}$ for $0 \leq j \leq N-2$ and $f^{N-1}(p) \in I_{f}$ for some $N \in \mathbb{N}$, otherwise $\mathcal{O}(p)=\left\{p, f(p), f^{2}(p), \ldots\right\}$. If $\mathcal{O}(p)$ is finite with $f^{N-1}(p) \in I_{f}$ then we say that $p$ has a singular orbit of length $N$; otherwise we say that it has a non-singular orbit.

A hypersurface $H$ is called exceptional if $\operatorname{dim} f\left(H-I_{f}\right)<d-1$. The only exceptional hypersurfaces of $f$ are of the form

$$
\Sigma_{i}:=\left\{\left[x_{0}: \cdots: x_{d}\right] \in \mathbb{P}^{d}: x_{i}=0\right\} .
$$

In fact $p_{i}:=f\left(\Sigma_{i}-I_{f}\right)$ is the $i^{t h}$ column of the matrix $L$. It follows from Theorem 5.3 that $f$ is 1 -regular if and only if $f^{n}\left(\Sigma_{i}-I_{f^{n}}\right) \not \subset I_{f}$ for every $n \geq 1$ and $i \in\{0,1, \ldots, d\}$. We denote the orbit of $\Sigma_{i}$ by $\mathcal{O}_{i}:=\mathcal{O}\left(p_{i}\right)$. Then it is easy to see that the orbit $\mathcal{O}_{i}$ is given by $p_{i, j}=\left[1: \cdots: 1: \frac{j\left(a_{i}-1\right)}{j a_{i}-(j-1)}: 1: \cdots: 1\right]$ for $j=1,2 \ldots$ and $\mathcal{O}_{i}$ is contained in the complex line $\mathcal{D}_{i}$. Thus, $\mathcal{O}_{i} \cap \mathcal{O}_{j}=\emptyset$ for $i \neq j$. In particular, $\mathcal{O}_{i}$ is singular if and only if $a_{i}=\frac{N-1}{N}$ for some $N \in \mathbb{N}_{+}$and in this 
case $f^{N-1}\left(p_{i}\right)=e_{i}:=[0: \cdots: 0: 1: 0 \cdots: 0]$ where 1 is in the $i^{\text {th }}$ component. Let $\mathcal{O}_{i}=\left\{p_{i, j}\right\}_{j=1}^{N_{i}}$ be a singular orbit then we denote its length by $\left|\mathcal{O}_{i}\right|:=N_{i}$. We define the set $S:=\left\{i: \mathcal{O}_{i}\right.$ is singular $\}$ and we also set $\mathcal{O}_{S}:=\bigcup_{i \in S} \mathcal{O}_{i}$. By conjugating $f$ with an involution, without lost of generality we may assume that $S=\{0,1, \ldots, k\}$ with $N_{0} \leq N_{1} \leq \cdots \leq N_{k}$ where $0 \leq k \leq d+1$ and we define $l$ by $l:=\#\left\{i \in S: a_{i}=0\right\}$ if the later set is non-empty otherwise we set $l=0$.

Let $\pi: X \rightarrow \mathbb{P}^{d}$ be the complex manifold obtained by blowing up the points in the set $\mathcal{O}_{S}$ successively. Then $f$ induces a birational map $f_{X}: X \rightarrow X$.

We denote the exceptional fiber over the point $p_{i, j} \in \mathcal{O}_{S}$ by $P_{i, j}:=\pi^{-1}\left(p_{i, j}\right)$. We also define the class $H_{X}:=\pi^{*} H$ where $H \subset \mathbb{P}^{d}$ is class of a generic hyperplane and let $P_{i, j}$ denote the class of exceptional divisor over $p_{i, j}$. Then $\left\{H_{X}, P_{0,1}, P_{0,2}, \ldots, P_{k, N_{k}}\right\}$ forms a basis for $H^{1,1}(X, \mathbb{R})$ and the action of $f_{X}^{*}$ on $H^{1,1}(X, \mathbb{R})$ is given by

$$
\begin{aligned}
f_{X}^{*}\left(H_{X}\right) & =d H_{X}-(d-1) \sum_{i \in S} P_{i, N_{i}} \\
f_{X}^{*}\left(P_{i, j+1}\right) & =P_{i, j} \text { for } 1 \leq j \leq N_{i}-1 \\
f_{X}^{*}\left(P_{i, 1}\right) & =\left\{\widetilde{\Sigma_{i}}\right\}=H_{X}-\sum_{\substack{j \in S \\
j \neq i}} P_{j, N_{j}}
\end{aligned}
$$

where $\widetilde{\Sigma_{j}} \subset X$ denotes the strict transform of $\Sigma_{j} \subset \mathbb{P}^{d}$ (see [ $[\overline{\mathrm{BK} 04}, \S 3]$ for details).

Theorem 6.1. BK04 Let $f_{X}: X \rightarrow X$ be as above then $f_{X}$ is 1-regular and characteristic polynomial of $f_{X}^{*}$ is given by

$$
\chi(x)=(x-1)^{l}\left[(x-(d-l)) \prod_{j=l}^{k}\left(x^{N_{j}}-1\right)+(x-1) \sum_{j=l}^{k} \prod_{\substack{i=l \\ i \neq j}}^{k}\left(x^{N_{i}}-1\right) .\right.
$$

Moreover, if $S \neq \emptyset$ and

$$
d-l \geq 3
$$

then $d-l-1 \leq \lambda:=\lambda_{1}(f) \leq d$ is the unique eigenvalue of $f_{X}^{*}$ of modulus greater than one and is a simple root of $\chi(x)$.

In the sequel, we will assume that $d \geq 3$ and $f_{X}$ is as in Theorem 6.1 so that (6.5) holds. We denote the corresponding eigenvector by $\alpha_{f} \in H^{1,1}(X, \mathbb{R})$ with $f_{X}^{*} \alpha_{f}=\lambda \alpha_{f}$ and we normalize it so that

$$
\alpha_{f}=H_{X}-c \cdot E
$$

where $c=\left(c_{0,1}, c_{0,2}, \ldots, c_{k, N_{k}}\right)$ and $E=\left(P_{0,1}, P_{0,2}, \ldots, P_{k, N_{k}}\right)$.

Lemma 6.2. Let $\alpha_{f}=H_{X}-c \cdot E$ be as above then for every $0 \leq i \leq k$

(1) $c_{i, j+1}=\lambda c_{i, j}$ for $1 \leq j \leq N_{i}-1$

(2) $\sum_{i=0}^{k} c_{i, 1}=d-\lambda$

(3) $c_{i, 1}=\frac{\lambda-1}{\lambda^{N_{i}-1}}>0$

(4) $\sum_{j=1}^{N_{i}} c_{i, j}=1$. 
Proof. (1) and (2) follows from the invariance of $\alpha_{f}$ and (6.2)-(6.4).

(3) For fixed $i$ we compare the coefficient of $P_{i, N_{i}}$ on the both sides of $f^{*} \alpha_{f}=\lambda \alpha_{f}$ and obtain

$$
(d-1)-\sum_{\substack{j=0 \\ j \neq i}}^{k} c_{j, 1}=\lambda c_{i, N_{i}}
$$

then by (1) and (2) we get

$$
c_{i, 1}=\frac{\lambda-1}{\lambda^{N_{i}}-1} .
$$

(4) follows from (1) and (3).

Proposition 6.3. The class $\alpha_{f} \in H_{n e f}^{1,1}(X, \mathbb{R})$ if and only if $|S| \leq 1$.

Proof. If $S=\emptyset$ then $X=\mathbb{P}^{d}$ and $\alpha_{f}=\left\{\omega_{F S}\right\}$ which is Kähler.

Assume that $|S|=1$. Then $\mathcal{O}_{0}$ is singular and the orbit is

$$
\Sigma_{0} \rightarrow p_{1} \rightarrow \cdots \rightarrow p_{N}=e_{0} .
$$

Let $H_{i} \subset \mathbb{P}^{d}$ denote a hyperplane such that $p_{i} \in H_{i}$ and $p_{j} \notin H_{i}$ for $j \neq i$. Notice that $H_{i}$ 's form a $(d-1)$-parameter family of hyperplanes. Since $\left\{\widetilde{H}_{i}\right\}=H_{X}-P_{i}$ by Lemma 6.2 we can represent the class $\alpha_{f}$ as the class of effective divisor $\sum_{i=1}^{N} c_{i} \widetilde{H}_{i}$ where $\widetilde{H_{i}}$ is the the strict transform of $H_{i}$. Since $\sum_{i=1}^{N} c_{i}\left[\widetilde{H}_{i}\right] \in \alpha_{f}$ defines a positive closed $(1,1)$ current, we infer that $\nu\left(T_{\alpha}^{\text {min }}, x\right)=0$ for every $x \in X$. Thus, it follows from Proposition 2.4 that $\alpha_{f}$ is nef.

Now, we will prove that if $|S| \geq 2$ then $\alpha_{f}$ is not nef. Indeed, let $\mathcal{O}_{i_{1}}$ and $\mathcal{O}_{i_{2}}$ be two singular orbits then by Lemma 6.2 and Theorem 6.1

$$
c_{i_{j}, N_{i_{j}}}>1-\frac{1}{\lambda} \geq 1-\frac{1}{d-l-1} \geq \frac{1}{2} \text { for } j=1,2 .
$$

Let $\ell$ denote the complex line passing through the points $e_{i_{1}}$ and $e_{i_{2}}$ and $\tilde{\ell}$ be its strict transform in $X$ then

$$
\alpha_{f} \cdot \tilde{\ell}=1-c_{i_{1}, N_{i_{1}}}-c_{i_{2}, N_{i_{2}}}<0
$$

hence by Proposition 2.9 we get $\tilde{\ell} \subset E_{n n}\left(\alpha_{f}\right)$.

Let $\Sigma_{I} \subset \mathbb{P}^{d}$ be as above, we also write $\Sigma_{I}$ for its strict transform inside $X$.

Proposition 6.4. If $1 \leq k \leq d-1$ and $2 \leq N:=N_{i}$ for every $0 \leq i \leq k$ then

$$
E_{n n}\left(\alpha_{f}\right)= \begin{cases}\Sigma_{\{k+1, \ldots, d\}} & \text { if } k \leq d-2, \\ \bigcup_{i=0}^{k} \Sigma_{\{i, d\}} & \text { if } k=d-1\end{cases}
$$

In particular, $1 \leq \operatorname{dim}_{\mathbb{C}} E_{n n}\left(\alpha_{f}\right) \leq(d-2)$ and $E_{n n}\left(\alpha_{f}\right) \subset I_{f_{X}}$ is algebraic.

Proof. It follows from Lemma 6.2 that $c_{i_{1}, l}=c_{i_{2}, l}$ for all $i_{1}, i_{2} \in S=\{0, \ldots, k\}$ and $1 \leq l \leq N$. We denote $c_{l}:=c_{i, l}$ for $i \in\{0, \ldots, k\}$.

If $|S|=2$ then $\Sigma_{\{2, \ldots, d\}}$ is a complex line and in the proof of Proposition 6.3 we have already showed that $\Sigma_{\{2, \ldots, d\}} \subset E_{n n}\left(\alpha_{f}\right)$.

Assume that $3 \leq|S|=k+1 \leq(d-1)$ and let $p \in \Sigma_{\{k+1, \ldots, d\}} \cong \mathbb{P}^{k}$ be a point. Let 
$\gamma \subset \Sigma_{\{k+1, \ldots, d\}}$ be an algebraic irreducible curve of degree $k$ such that $p, e_{i} \in \gamma$ for every $0 \leq i \leq k$. Then by Lemma 6.2 and by (6.5) we have $c_{N}>1-\frac{1}{d-1}$ and

$$
\alpha_{f} \cdot \gamma=k-(k+1) c_{N}<\frac{k+1}{d-1}-1 \leq 0 .
$$

Thus, by Proposition 2.9 we get $\Sigma_{\{k+1, \ldots, d\}} \subset E_{n n}\left(\alpha_{f}\right)$.

If $k=d-1$ we can apply the same argument to $\{0, \ldots, d-1\}-\{i\}$ for $0 \leq i \leq$ $d-1$.

To prove the reverse inclusion we will represent the class $\alpha_{f}$ by effective divisors: Notice that each $p_{i, l}=\left[1: \cdots: 1: \frac{l\left(a_{i}-1\right)}{l a_{i}-(l-1)}: 1: \cdots: 1\right] \in \mathcal{D}_{i}$ which is a complex line. Let $H_{l} \subset \mathbb{P}^{d}$ be a hyperplane such that $p_{i, l} \in H_{l}$ for every $0 \leq i \leq k$ and $p_{i, m} \notin H_{l}$ for $m \neq l$. This is a $d-k-1$ parameter family of hyperplanes for each $l$. Then, the class $\left\{\widetilde{H}_{l}\right\}=H_{X}-\sum_{i=0}^{k} P_{i, l}$ where $\widetilde{H}_{l}$ denotes the strict transform of $H_{l}$. Hence, by Lemma 6.2 we can represent $\alpha_{f}$ by

$$
\alpha_{f}=\sum_{l=1}^{N} c_{l}\left\{\widetilde{H}_{l}\right\}
$$

Next, we assume that $k \leq d-2$. We consider the hyperplanes of the form $D_{i}=\left\{x \in \mathbb{P}^{d}: 2 x_{i}-x_{d-1}-x_{d}=0\right\}$ where $0 \leq i \leq k$ is fixed. Then the complex line $\mathcal{D}_{j} \subset D_{i}$ for $0 \leq j \neq i \leq k$ and $\mathcal{O}_{i} \cap D_{i}=\emptyset$. Thus, $\left\{\widetilde{D_{i}}\right\}=H_{X}-\sum_{\substack{l=1 \\ 0 \leq j \neq i \leq k}}^{N} P_{j, l}$.

We also denote $H_{\Sigma_{\{k+1, \ldots, d\}}} \subset \mathbb{P}^{d}$ be a hyperplane containing $\Sigma_{\{k+1, \ldots, d\}}$ such that $\left\{H_{\Sigma_{\{k+1, \ldots, d\}}}\right\}=H_{X}-\sum_{i \in S} P_{i, N}$ (Eg. $H_{\Sigma_{\{k+1, \ldots, d\}}}=\Sigma_{j}$ for some $k+1 \leq j \leq d$ ). Then by Lemma 6.2

$$
\alpha_{f}=\sigma \sum_{i=0}^{k}\left\{\widetilde{D}_{i}\right\}+(1-\sigma(k+1))\left\{\widetilde{H}_{\Sigma_{\{k+1, \ldots, d\}}}\right\}+\mathcal{E}
$$

where $\sigma=1-c_{N}$ and $\mathcal{E}$ is an effective divisor supported on $\underset{\substack{i \in\{0, \ldots, k\} \\ 1 \leq l \leq N-1}}{\bigcup} P_{i, l}$. Indeed,

it follows from Lemma 6.2 that

$$
1-\sigma(k+1)=\frac{d-k-1}{\lambda}>0
$$

On the other hand, we can also represent $\alpha_{f}$ as follows: let $i_{1}, i_{2} \in S$ then

$$
\alpha_{f}=\sigma\left(\left\{\widetilde{D_{i_{1}}}\right\}+\left\{\widetilde{D_{i_{2}}}\right\}\right)+(1-2 \sigma)\left\{\widetilde{H}_{\Sigma_{\{k+1, \ldots, d\}}}\right\}+\mathcal{E}^{\prime}
$$

where $\mathcal{E}^{\prime}$ is an effective divisor supported on $\bigcup_{\substack{i \in\{0, \ldots, k\} \\ 1 \leq l \leq N}} P_{i, l}$ and $\sigma$ is as above. Since the non-nef locus is contained in the intersection of the supports of the effective divisors in (6.6), (6.7) and (6.8) we conclude that

$$
E_{n n}\left(\alpha_{f}\right) \subset \Sigma_{\{k+1, \ldots, d\}} .
$$

If $k=d-1$ then we claim that $c_{N}=\frac{d-1}{d}$. Indeed, by Lemma 6.2 (2) $c_{1}=\frac{(d-\lambda)}{d}$ and by using Lemma 6.2 (3) we get $\lambda^{N-1}=\frac{d-1}{d-\lambda}$. Then by Lemma 6.2(1) we get 
$c_{N}=\lambda^{N-1} c_{1}=\frac{d-1}{d}$. This implies that we can represent $\alpha_{f}$ as

$$
\alpha_{f}=\frac{1}{d} \sum_{i=0}^{d-1}\left\{\widetilde{L_{i}}\right\}+\mathcal{E}
$$

where $L_{i}:=\left\{x \in \mathbb{P}^{d}: x_{i}-x_{d}=0\right\}$ and $\mathcal{E}$ is an effective divisor supported on $\bigcup P_{i, l}$. Now, for fixed $0 \leq j \leq d-1$ we also have $1 \leq l \leq N-1$

$$
\alpha_{f}=\frac{1}{d} \sum_{\substack{i=0 \\ i \neq j}}^{d-1}\left\{\widetilde{L_{i}}\right\}+\frac{1}{d}\left\{\widetilde{F_{j}}\right\}+\mathcal{E}^{\prime}
$$

where $F_{j}:=\left\{x \in \mathbb{P}^{d}: x_{j}-a x_{d}=0\right\}$ is a 1-parameter family of hyperplanes and $\mathcal{E}^{\prime}$ is an effective divisor supported on $\bigcup_{\substack{0 \leq i \leq d-1 \\ 1 \leq l \leq N-1}} P_{i, l}$. Hence, by (6.6), (6.9) and (6.10) we get

$$
E_{n n}\left(\alpha_{f}\right) \subset \bigcup_{i=0}^{d-1} \Sigma_{\{i, d\}} .
$$

Now, we prove that a generic mapping of the form $f=L \circ J$ fall into framework of Theorem 1.2 ,

Proof of Theorem 1.5. If $|S| \leq 1$ then the assertion follows from Proposition 6.3 and Theorem 4.4

We assume that $|S| \geq 2$ and set $S=\{0, \ldots, k\}$. By Theorem 5.6 and Corollary 5.7 it is enough to show that $\alpha_{f} \cdot C \geq 0$ for every algebraic irreducible curve $C \subset E_{f_{X}}^{-}$. Since $f_{X}$ is biholomorphic near the exceptional fibers $P_{i, j}$ 's, the indeterminacy locus is given by

$$
I_{f_{X}}=\bigcup_{|I| \geq 2} \widetilde{\Sigma_{I}}
$$

This implies that $f_{X}\left(I_{f_{X}}\right) \subset \bigcup_{i=0}^{d} L\left(\widetilde{\Sigma_{i}}\right)$ where $L\left(\Sigma_{i}\right)=\left\{x \in \mathbb{P}^{d}: a \cdot x-x_{i}=0\right\}$ and $a=\left[a_{0}: \cdots: a_{d}\right]$. Since $p_{i, j}=\left[1: \cdots: \frac{j\left(a_{i}-1\right)}{j a_{i}-(j-1)}: \cdots: 1\right]$ we infer that

$$
\bigcup_{i=0}^{d} L\left(\Sigma_{i}\right) \cap \mathcal{O}_{S}=\left\{p_{0,1}, p_{1,1}, \ldots, p_{k, 1}\right\} .
$$

Then for any algebraic irreducible curve $C \subset E_{f_{X}}^{-}$by Lemma 6.2

$$
\begin{aligned}
\alpha \cdot C & \geq \operatorname{deg} C-\sum_{i=0}^{k} c_{i, 1}\left(\text { mult }_{p_{i, 1}} C\right) \\
& \geq \operatorname{deg} C(1-(d-\lambda)) \\
& \geq 0
\end{aligned}
$$

where the last inequality follows from Theorem 6.1. 
Theorem 6.5. Let $f: \mathbb{P}^{3} \rightarrow \mathbb{P}^{3}$ be as above. If $|S| \leq 3$ and $2 \leq N:=N_{i}$ for every $i \in S$ then there exists a birational model $\mu: Y \rightarrow \mathbb{P}^{3}$ such that $f_{Y}$ : $Y \rightarrow Y$ is a dominant 1-regular map with $\lambda:=\lambda_{1}\left(f_{Y}\right)$ is the unique simple eigenvalue of modulus greater than 1 with the corresponding normalized eigenvector $\widetilde{\alpha_{f}} \in H_{n e f}^{1,1}(Y, \mathbb{R})$.

Proof. If $|S| \leq 1$ then the assertion follows from Theorem 6.1 and Proposition 6.3. We assume that $S=\{0,1\}$ then we define the complex manifold $Y$ to be $X$ blown up along $E_{n n}\left(\alpha_{f}\right)=\Sigma_{23}$ which is a complex line. We denote the projection by $\mu: Y \rightarrow X$ and the exceptional divisor by $\mathcal{F}:=\mu^{-1}\left(E_{n n}\left(\alpha_{f}\right)\right)$.

We first show that the induced map $f_{Y}: Y \rightarrow Y$ is 1-regular: Notice that the only exceptional hypersurfaces of $f_{Y}$ are $\widetilde{\Sigma}_{i}$ for $i \notin S$ and $\mathcal{F}$. Since $f_{Y}^{n}\left(\widetilde{\Sigma}_{i}-I_{f^{n}}\right) \not \subset I_{f_{Y}}$ for $n \geq 1$ and $i \notin S$ by Theorem 5.3 it's enough to check that $f_{Y}^{n}(\mathcal{F}) \not \subset I_{f_{Y}}$ for every $n \in \mathbb{N}$.

We claim that $\overline{f_{Y}\left(\mathcal{F} \backslash I_{f_{Y}}\right)}=\widetilde{L\left(\Sigma_{S}\right)}$. Indeed, we write $f_{Y}$ in the local coordinates: $\left(\eta_{1}, \eta_{2}, s\right) \in Y$ where $\mathcal{F}=\{s=0\}$ and

$$
\begin{aligned}
\pi_{Y} & : Y \rightarrow \mathbb{P}^{3} \\
\mu\left(\eta_{1}, \eta_{2}, s\right) & =\left[1: \eta_{1}: \eta_{2} s: s\right]
\end{aligned}
$$

Then, we may identify

$$
f_{Y}\left(\eta_{1}, \eta_{2}, 0\right)=\eta_{1}\left[a_{2}: a_{2}: a_{2}-1: a_{2}\right]+\eta_{1} \eta_{2}\left[a_{3}: a_{3}: a_{3}: a_{3}-1\right]
$$

which proves the claim. Since the points $\left[a_{2}: a_{2}: a_{2}-1: a_{2}\right]$ and $\left[a_{3}: a_{3}: a_{3}: a_{3}-1\right]$ have non-singular orbits we conclude that $f_{Y}$ is 1-regular. Similarly, one can show that $\overline{f_{Y}^{-1}\left(\mathcal{F} \backslash I_{f_{Y}^{-1}}\right)}=J\left(\operatorname{span}\left\{\left[a_{0}-1: a_{0}: a_{0}: a_{0}\right],\left[a_{1}: a_{1}-1: a_{1}: a_{1}\right]\right\}\right)$ where the later set has codimension 2 .

Now, $\left\{H_{Y}, P_{0,1}, P_{0,2}, . ., P_{1, N}, \mathcal{F}\right\}$ forms an ordered basis for $H^{1,1}(X, \mathbb{R})$ where $H_{Y}:=\mu^{*}\left(H_{X}\right)$ and $P_{i, l}:=\mu^{*}\left(P_{i, l}\right)$ for each $i, 1 \leq l \leq N$ and the action of $f_{Y}^{*}: H^{1,1}(Y) \rightarrow H^{1,1}(Y)$ is given by

$$
\begin{aligned}
f_{Y}^{*}\left(H_{Y}\right) & =3 H_{X}-2 P_{0, N}-2 P_{1, N}-\mathcal{F} \\
f_{Y}^{*}\left(P_{i, l+1}\right) & =P_{i, l} \text { for } 1 \leq l \leq N-1 \text { and } i \in S \\
f_{Y}^{*}\left(P_{i, 1}\right) & =\widetilde{\Sigma_{i}}, \text { for } i \in S \\
f_{Y}^{*}(\mathcal{F}) & =0
\end{aligned}
$$

where $\widetilde{\Sigma_{i}} \subset Y$ denotes the strict transform of $\Sigma_{i} \subset \mathbb{P}^{3}$. Thus, the characteristic polynomial of $f_{Y}^{*}$ is given by $p(x)=x \chi(x)$ where $\chi(x)$ is as in Theorem 6.1. This implies that $\lambda=\lambda_{1}\left(f_{Y}\right)$ is a simple eigenvalue. Moreover, corresponding eigenvector $\widetilde{\alpha_{f}}$ is of the form

$$
\widetilde{\alpha_{f}}=H_{Y}-c \cdot E-\frac{1}{\lambda} \mathcal{F}
$$

where $\mathrm{c}$ and $\mathrm{E}$ are as in Lemma 6.2

Now, we claim that $\widetilde{\alpha_{f}}$ is nef. Indeed, it follows from Lemma 6.2 that

$$
1-2 \sigma=\frac{1}{\lambda}
$$

and by the representations (6.6), (6.7) and (6.8) we infer that $\nu\left(T_{\overline{\alpha_{f}}}^{\min }, y\right)=0$ for every $y \in Y$. Hence, the claim follows.

If $|S|=3$ then by Proposition $6.4 E_{n n}\left(\alpha_{f}\right)$ has 3 components which are pairwise 
disjoint complex lines in $X$. In this case, we define the complex manifold $Y$ to be $X$ blown up along each component of $E_{n n}\left(\alpha_{f}\right)$ successively and apply the above analysis to drive the assertion. We omit the details of this part.

\section{REFERENCES}

[BK04] E. Bedford and K. Kim. On the degree growth of birational mappings in higher dimension. J. Geom. Anal., 14(4):567-596, 2004.

[BS92] E. Bedford and J. Smillie. Polynomial diffeomorphisms of $\mathbf{C}^{2}$. III. Ergodicity, exponents and entropy of the equilibrium measure. Math. Ann., 294(3):395-420, 1992.

[Bou04] S. Boucksom. Divisorial Zariski decompositions on compact complex manifolds. Ann. Sci. École Norm. Sup. (4), 37(1):45-76, 2004.

[BDPP] S. Boucksom, J.-P. Demailly, M. Paun, and T. Peternell. The pseudo-effective cone of a compact kähler manifold and varieties of negative kodaira dimension,arxiv:math/0405285, 2004.

[BHM03] S. Boukraa, S. Hassani, and J.-M. Maillard. Noetherian mappings. Phys. D, 185(1):3-44, 2003.

[Dem92] J.-P. Demailly. Regularization of closed positive currents and intersection theory. $J$. Algebraic Geom., 1(3):361-409, 1992.

[DP04] J.-P. Demailly and M. Paun. Numerical characterization of the Kähler cone of a compact Kähler manifold. Ann. of Math. (2), 159(3):1247-1274, 2004.

[DPS01] J.-P. Demailly, T. Peternell, and M. Schneider. Pseudo-effective line bundles on compact Kähler manifolds. Internat. J. Math., 12(6):689-741, 2001.

[DDG10] J. Diller, R. Dujardin, and V. Guedj. Dynamics of meromorphic maps with small topological degree i: from cohomology to currents. Indiana Univ. Math. J., 59:521-562, 2010.

[DF01] J. Diller and C. Favre. Dynamics of bimeromorphic maps of surfaces. Amer. J. Math., 123(6):1135-1169, 2001.

[DG09] J. Diller and V. Guedj. Regularity of dynamical Green's functions. Trans. Amer. Math. Soc., 361(9):4783-4805, 2009.

[DS04] T.-C. Dinh and N. Sibony. Regularization of currents and entropy. Ann. Sci. École Norm. Sup. (4), 37(6):959-971, 2004.

[DS05] T.-C. Dinh and N. Sibony. Green currents for holomorphic automorphisms of compact Kähler manifolds. J. Amer. Math. Soc., 18(2):291-312 (electronic), 2005.

[DS08] T.-C. Dinh and N. Sibony. Equidistribution towards the Green current for holomorphic maps. Ann. Sci. Éc. Norm. Supér. (4), 41(2):307-336, 2008.

[Fav00] C. Favre. Multiplicity of holomorphic functions. Math. Ann., 316(2):355-378, 2000.

[FG01] C. Favre and V. Guedj. Dynamique des applications rationnelles des espaces multiprojectifs. Indiana Univ. Math. J., 50(2):881-934, 2001.

[FS95] J. E. Fornaess and N. Sibony. Complex dynamics in higher dimension. II. In Modern methods in complex analysis (Princeton, NJ, 1992), volume 137 of Ann. of Math. Stud., pages 135-182. Princeton Univ. Press, Princeton, NJ, 1995.

[GH78] P. Griffiths and J. Harris. Principles of algebraic geometry. Wiley-Interscience [John Wiley \& Sons], New York, 1978. Pure and Applied Mathematics.

[Gue02] V. Guedj. Dynamics of polynomial mappings of $\mathbb{C}^{2}$. Amer. J. Math., 124(1):75-106, 2002.

[Gue04] V. Guedj. Decay of volumes under iteration of meromorphic mappings. Ann. Inst. Fourier (Grenoble), 54(7):2369-2386 (2005), 2004.

[Gue] V. Guedj. Propriétés ergodiques des applications rationelles, arxiv:math/0611302, 2006.

[HP75] R. Harvey and J. Polking. Extending analytic objects. Comm. Pure Appl. Math., 28(6):701-727, 1975.

[Huc94] A. Huckleberry. Subvarieties of homogeneous and almost homogeneous manifolds. In Contributions to complex analysis and analytic geometry, Aspects Math., E26, pages 189-232. Vieweg, Braunschweig, 1994.

[Kis00] C. O. Kiselman. Ensembles de sous-niveau et images inverses des fonctions plurisousharmoniques. Bull. Sci. Math., 124(1):75-92, 2000.

[KM98] J. Kollár and S. Mori. Birational geometry of algebraic varieties, volume 134 of Cambridge Tracts in Mathematics. Cambridge University Press, Cambridge, 1998. With 
GREEN CURRENTS FOR MEROMORPHIC MAPS OF COMPACT KÄHLER MANIFOLDS 25

the collaboration of C. H. Clemens and A. Corti, Translated from the 1998 Japanese original.

[Meo96] M. Meo. Image inverse d'un courant positif fermé par une application analytique surjective. C. R. Acad. Sci. Paris Sér. I Math., 322(12):1141-1144, 1996.

[Ngu] V.-A. Nguyen. Green currents for quasi-algebraically stable meromorphic self-maps of $\mathbb{C} P^{k}$,arxiv:0902.1376v2, 2011.

[Sib99] N. Sibony. Dynamique des applications rationnelles de $\mathbf{P}^{k}$. In Dynamique et géométrie complexes (Lyon, 1997), volume 8 of Panor. Synthèses, pages ix-x, xi-xii, 97-185. Soc. Math. France, Paris, 1999.

Mathematics Department, Indiana University 47405 Indiana, USA

E-mail address: tbayrakt@indiana.edu 